\title{
Brauer groups of moduli of hyperelliptic curves via cohomological invariants
}

\author{
Andrea Di Lorenzo ${ }^{(\mathbb{D})}$ and Roberto Pirisi ${ }^{\left(D_{2}\right.}$ \\ ${ }^{1}$ Department of Mathematics, Humboldt-Universität zu Berlin, Unter den Linden 6, Berlin 10099, Germany; \\ E-mail: andrea.dilorenzo@hu-berlin.de. \\ ${ }^{2}$ Dipartimento di Matematica Guido Castenuovo, Sapienza Università di Roma, Piazzale Aldo Moro 5, Rome, 00185, Italy; \\ E-mail: roberto.pirisi@uniroma1.it.
}

Received: 15 March 2021; Revised: 29 June 2021; Accepted: 3 August 2021

Keywords: Brauer Group, moduli stack, hyperelliptic curves, cohomological invariants

2020 Mathematics Subject Classification Codes: Primary - 14F22; Secondary - 14H10

\begin{abstract}
Using the theory of cohomological invariants for algebraic stacks, we compute the Brauer group of the moduli stack of hyperelliptic curves $\mathcal{H}_{g}$ over any field of characteristic 0 . In positive characteristic, we compute the part of the Brauer group whose order is prime to the characteristic of the base field.
\end{abstract}

\section{Contents}

1 Introduction $\quad \mathbf{2}$

1.1 Brauer groups of moduli stacks . . . . . . . . . . . . . . . . . 2

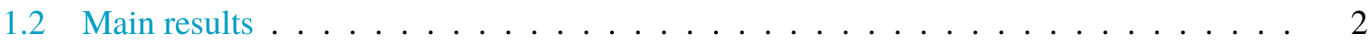

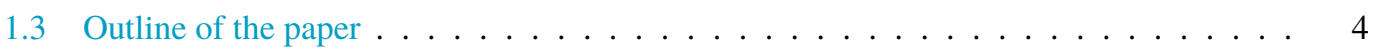

1.4 Notation . . . . . . . . . . . . . . . . . . . . . . 4

2 Preliminaries 4

2.1 Brauer group, cohomological Brauer group, cyclic algebras . . . . . . . . . . . . . . 4

2.2 Generalised cohomological invariants . . . . . . . . . . . . . . . 6

2.3 Cohomological invariants and the cohomological Brauer group . . . . . . . . . . . . 10

2.4 Chow groups with coefficients . . . . . . . . . . . . . . . 12

3 The Brauer group of $\mathcal{M}_{1,1} \quad \mathbf{1 5}$

4 Some equivariant computations $\quad 16$

5 Generalised cohomological invariants of some classical groups $\quad 22$

6 The moduli stacks of hyperelliptic curves $\quad 26$

7 The generalised cohomological invariants of $\mathcal{H}_{g} \quad \mathbf{2 8}$

8 The Brauer group of $\mathcal{H}_{g} \quad 35$

Acknowledgements $\quad 36$

$\begin{array}{lr}\text { References } & 37\end{array}$

(C) The Author(s), 2021. Published by Cambridge University Press. This is an Open Access article, distributed under the terms of the Creative Commons Attribution licence (http://creativecommons.org/licenses/by/4.0/), which permits unrestricted re-use, distribution, and reproduction in any medium, provided the original work is properly cited. 


\section{Introduction}

\subsection{Brauer groups of moduli stacks}

Brauer groups of fields have long been an object of study in number theory, dating back to work of Noether and Brauer. They were later generalised by Grothendieck to schemes and more general objects, up to the vast generality of topoi.

While Brauer groups of schemes have seen a lot of attention in modern algebraic geometry, computations of Brauer groups of moduli stacks over nonalgebraically closed fields only started appearing in recent years.

In the 2010s, Lieblich [19] computed the Brauer group of $\mathrm{B} \mu_{q}$ over fields where $q$ is invertible and applied it to the period-index problem. Later, Antieau and Meier [1] computed the Brauer group of the moduli stack $\mathcal{M}_{1,1}$ of elliptic curves over a variety of bases, including $\mathbb{Z}, \mathbb{Q}$ and any finite field of characteristic greater than 2. Moreover, in an unpublished draft [20], Meier computes the $\ell$-torsion of the Brauer group of $\mathcal{M}_{1,1}$ over any separated, regular noetherian scheme $S$ where $\ell$ is invertible.

In 2019, Shin [26] showed that over an algebraically closed field of characteristic 2, the Brauer group of $\mathcal{M}_{1,1}$ is equal to $\mathbb{Z} / 2 \mathbb{Z}$.

The proofs of these results are based on standard tools in étale and flat cohomology coupled with a very delicate analysis of various presentations of the stack of elliptic curves, their relations, the stabiliser groups at various points and so on, which seem hard to apply to more complicated stacks.

\subsection{Main results}

Our goal is twofold:

1. Introducing a new toolkit for the computation of Brauer groups of moduli stacks, based on the second author's theory of cohomological invariants for algebraic stacks [22]

2. Computing the prime-to-char $(k)$ part of $\operatorname{Br}\left(\mathcal{H}_{g}\right)$, the Brauer group of the moduli stacks of hyperelliptic curves of genus $g \geq 2$, over a field $k$ of characteristic different from 2 (see Main Theorem A); in particular, if $\operatorname{char}(k)=0$ we obtain the full Brauer group

To simplify our statements, we introduce the following notation:

Definition. Let $A$ be an abelian torsion group and let $c$ be a prime number or 0 . We denote by ${ }^{c} A$ the subgroup of $A$ given by elements whose order is not divisible by $c$. In particular, ${ }^{0} A=A$. Given a positive number $\ell$, we denote by $A_{\ell}$ the $\ell$-tosion subgroup.

Without further ado, we state our main Theorem (Theorem 8.1):

Main Theorem A. Let $k$ be a field of characteristic $c \neq 2$ and $g>1$ an integer. Let $r_{g} \in\{0,1\}$ be the remainder of $g$ mod 2 . Let $\ell_{g}=2^{r_{g}}(4 g+2)$.

Then

$$
{ }^{c} \operatorname{Br}\left(\mathcal{H}_{g}\right) \simeq{ }^{c} \operatorname{Br}(k) \oplus \mathrm{H}_{\mathrm{Gal}}^{1}\left(k,{ }^{c}\left(\mathbb{Z} / \ell_{g} \mathbb{Z}\right)\right) \oplus \mathbb{Z} / 2 \mathbb{Z}^{\oplus\left(1+r_{g}\right)}
$$

Note that while in Antieau and Meier's results the Brauer group is generated by the Brauer group of the base field and cyclic algebras, in our case the $\mathbb{Z} / 2 \mathbb{Z}^{\oplus\left(1+r_{g}\right)}$ component does not, painting a richer picture. The additional copy of $\mathbb{Z} / 2 \mathbb{Z}$ in the odd case is generated by the class of the relative BrauerSeveri scheme $P=\mathcal{C} / \iota$, where $\mathcal{C}$ is the universal curve over $\mathcal{H}_{g}$ and $\iota$ is the hyperelliptic involution. It is well known that this class is trivial when $g$ is even.

As we mentioned earlier, our techniques are based on the second author's theory of cohomological invariants for algebraic stacks [22] and computations in Rost's (equivariant) Chow groups with coefficients $[17,24]$. We believe these techniques will be well suited to computing the Brauer groups of a large variety of stacks admitting a 'good' quotient presentation, such as stacks of trigonal curves [3] 
and stacks that can be obtained as stacks of complete intersections - for example, the stacks of quasipolarised K3 surfaces of low degree [7].

In terms of computing cohomological invariants, our main result (combining Proposition 7.5, Theorem 7.9 and Remark 7.10) is the following:

Main Theorem B. Let $k$ be a field of characteristic $c \neq 2$, and let $\mathrm{M}$ be an $\ell$-torsion cycle module, with $c \nmid \ell$. There are elements $\alpha_{1}, \ldots, \alpha_{g+1}$, of degree $1, \ldots, g+1$, such that

$$
I_{g}=\mathbf{M}^{\bullet}(k) \oplus \alpha_{1} \cdot \mathbf{M}^{\bullet}(k)_{\ell_{g}} \oplus \bigoplus_{i=2}^{g+1} \alpha_{i} \cdot \mathbf{M}^{\bullet}(k)_{2}
$$

is a submodule of $\operatorname{Inv}^{\bullet}\left(\mathcal{H}_{g}, \mathrm{M}\right)$. If $g$ is even, we have

$$
\operatorname{Inv} \bullet\left(\mathcal{H}_{g}^{k}, \mathrm{M}\right)=I_{g} \oplus \beta_{g+2} \cdot \mathrm{M}^{\bullet}(k)_{2} .
$$

If $g$ is odd, there is an exact sequence

$$
0 \rightarrow I_{g} \oplus w_{2} \cdot \mathrm{M}^{\bullet}(k)_{2} \rightarrow \operatorname{Inv}\left(\mathcal{H}_{g}^{k}, \mathrm{M}\right) \rightarrow \mathrm{N}_{g}^{\bullet}(k) \rightarrow 0,
$$

where $w_{2}$ has degree $2, \mathrm{~N}_{g}^{\bullet}(k) \subseteq \mathrm{M}^{\bullet}(k)_{2}$ and the last map lowers degree by $g+2$.

The cohomological invariants in the theorem have coefficients in an arbitrary $\ell$-torsion cycle module $\mathrm{M}$, where $\ell$ is a positive integer not divided by $c$. An $\ell$-torsion cycle module admits a module structure over the cycle module $\mathrm{H}_{\mathbb{Z} / \ell \mathbb{Z}}$ of twisted $\bmod \ell$ étale cohomology, which has a natural graded-commutative

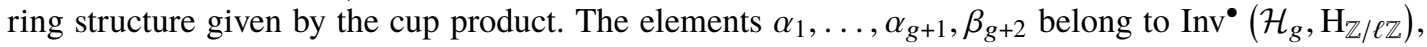
and the product is given by the module structure. Note that an arbitrary cycle module $\mathrm{M}$ may not have a ring structure. Consequently, different from our previous results, the group $\operatorname{Inv} \bullet^{\bullet}\left(\mathcal{H}_{g}, M\right)$ does not in general have a ring structure.

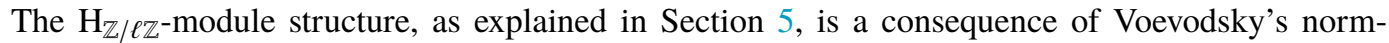
residue principle [29], also known as the Bloch-Kato conjecture. The norm-residue principle will be

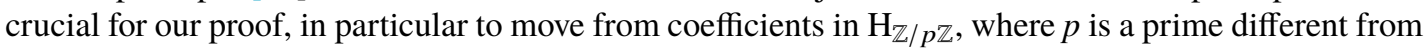

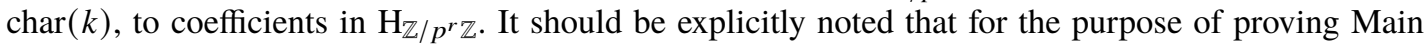
Theorem A, we can restrict to invariants of degrees 1 and 2 and thus use the much older and simpler Merkurjev-Suslin theorem.

Main Theorem B represents a large increase in generality compared to our previous results. In $[6,21,23]$, the foregoing result is obtained under the assumption that the base field is algebraically closed and the coefficients are taken in twisted $\bmod p$ étale cohomology, where $p$ is a prime number different from $c$. In [8], we managed to lift the condition that $k$ should be algebraically closed, but still used $\bmod p$ étale cohomology as coefficients.

The technical improvement between the present result and our previous results is composed of two

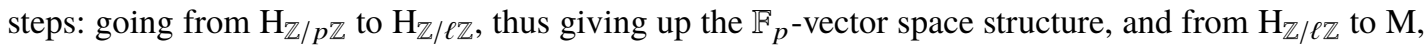
thus giving up the ring structure. The second step is the hardest, as the modules that appear are not very well behaved, not being free or faithful or even necessarily finitely generated. Nonetheless, as the formula in Main Theorem B shows, they will still admit reasonable decompositions in terms of the

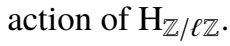

Dealing with more general cycle modules is necessary to obtain Brauer groups, as we need to consider twisted étale cohomology with coefficients in $\mathbb{Z} / \ell \mathbb{Z}(-1)=\mu_{\ell}^{\vee}$. Note in particular that $\mathrm{H}_{\mu_{\ell}^{\vee}}$ does not have a ring structure. We could restrict ourselves to just considering the cycle modules $\mathrm{H}_{D}$ coming from twisted étale cohomology with coefficients in a Galois module $D$, but extending the results to all torsion cycle modules (and in fact, for much of the paper, to all cycle modules) requires little extra effort.

As part of our supporting results, we obtain a slight sharpening of recent results by Gille and Hirsch [16] on classical cohomological invariants (with generalised coefficients) which might be of interest by itself. 


\subsection{Outline of the paper}

In Section 2 we establish the basic results we will need for the rest of the paper on Brauer groups, cohomological invariants and Chow groups with coefficients. In particular, in Subsection 2.3 we prove that the cohomological Brauer group of a quotient stack is computed by cohomological invariants.

Section 3 contains a first demonstration of our techniques. We extend a computation from [22] to compute the cohomological invariants with arbitrary coefficients of the stack of elliptic curves $\mathcal{M}_{1,1}$ and use it to compute its Brauer group, partially retrieving Antieau and Meier's result [1].

In Section 4 we compute the equivariant Chow groups with coefficients of various classifying stacks $\mathrm{B} G$, which will be used in our main computation.

Section 5 is dedicated to computing the generalised cohomological invariants of $\mathrm{BS}_{n}$ and $\mathrm{BPGL}_{2}$. We use Gille and Hirsch's splitting principle [16], complementing it with some equivariant computations which show that in every case where the splitting principle applies, any normalised cohomological invariant is of 2-torsion.

In Section 6 we describe a presentation of $\mathcal{H}_{g}$ by Arsie and Vistoli [2] and restate some results on the relation between the cohomological invariants of $\mathcal{H}_{g}$ and $\mathrm{BS}_{2 g+2}$ from [8].

Section 7 is where we put all the results together to obtain our computation of the cohomological invariants of $\mathcal{H}_{g}$.

Finally, in Section 8 we specialise the computation of cohomological invariants to obtain a presentation of the Brauer group, and we describe each generator.

\subsection{Notation}

We work over a base field $k$ of characteristic $c \neq 2$. The notation $\ell$ will be reserved for a positive integer, not necessarily prime, that is not divisible by $c$.

Every scheme and algebraic (also known as Artin) stack is assumed to be of finite type over $\operatorname{Spec}(k)$. By a Galois module over $k$ we always mean a locally constant sheaf of abelian groups on the small étale site of $\operatorname{Spec}(k)$.

Unless otherwise stated, by $\mathrm{H}^{i}(X, F)$ we always mean étale cohomology, or lisse-étale cohomology for algebraic stacks. If $R$ is a $k$-algebra, we will write $\mathrm{H}^{i}(R, F)$ for $\mathrm{H}^{i}(\operatorname{Spec}(R), F)$. Given a graded abelian group $A$, we denote by $A_{\ell}$ the $\ell$-torsion subgroup and by $A[d]$ the group shifted in degree by $d$.

\section{Preliminaries}

\subsection{Brauer group, cohomological Brauer group, cyclic algebras}

Given a Noetherian scheme $X$, the Brauer group $\operatorname{Br}(X)$ is the group of Azumaya algebras over $X-$ that is, sheaves of unitary algebras which are étale locally isomorphic to the endomorphism group of a vector bundle over $X$, modulo the relation that $\mathcal{E} \sim \mathcal{E}^{\prime}$ if there exist vector bundles $V$ and $V^{\prime}$ such that $\mathcal{E} \otimes \operatorname{End}(V) \simeq \mathcal{E}^{\prime} \otimes \operatorname{End}\left(V^{\prime}\right)$. This relation corresponds to Morita equivalence, and the group operation is given by tensor product.

The rank of an Azumaya algebra is always a square $n^{2}$, and algebras of rank $n^{2}$ are classified by $\mathrm{PGL}_{n}$-torsors, with the trivial ones coming from $\mathrm{GL}_{n}$-torsors. We have an exact sequence

$$
1 \rightarrow \mathbb{G}_{m} \rightarrow \mathrm{GL}_{n} \rightarrow \mathrm{PGL}_{n} \rightarrow 1
$$

which induces an exact sequence

$$
\mathrm{H}_{\text {êt }}^{1}\left(X, \mathrm{GL}_{n}\right) \rightarrow \mathrm{H}_{\text {êt }}^{1}\left(X, \mathrm{PGL}_{n}\right) \rightarrow \mathrm{H}_{\text {ét }}^{2}\left(X, \mathbb{G}_{m}\right) .
$$

The class of an Azumaya algebra always maps to a torsion element of $\mathrm{H}_{\text {ét }}^{2}\left(X, G_{m}\right)$, and the map is injective, so that $\operatorname{Br}(X) \subseteq \mathrm{H}^{2}\left(X, \mathbb{G}_{m}\right)_{\text {tor }}$. We call the torsion subgroup $\mathrm{H}_{\text {ét }}^{2}\left(X, \mathbb{G}_{m}\right)_{\text {tor }}$ the cohomological 
Brauer group of $X$, denoted $\operatorname{Br}^{\prime}(X)$. In the setting of schemes, due to results of Gabber and de Jong $[14,4]$, we know that $\operatorname{Br}(X)=\operatorname{Br}^{\prime}(X)$ whenever $X$ carries an ample line bundle.

The definition of the Brauer group can be vastly extended. Given an algebraic stack, one can define the Brauer group $\operatorname{Br}(\mathcal{X})$, and if $\mathcal{X}$ is quasi-compact or connected, the inclusion $\operatorname{Br}(\mathcal{X}) \subseteq \operatorname{Br}^{\prime}(\mathcal{X})$ holds (note that for algebraic stacks we will have to use lisse-étale cohomology).

The cohomological Brauer group is often easier to compute, and in all of our computations we will work with it and then check a posteriori that every element we find comes from an Azumaya algebra. One very important type of element that we know always come from the Brauer groups are those given by cyclic algebras.

First, note that if $\ell$ is prime to $\operatorname{char}(k)$, and $\mathcal{X}$ is an algebraic stack, then due to the Kummer exact sequence

$$
1 \rightarrow \mu_{\ell} \rightarrow \mathbb{G}_{m} \rightarrow \mathbb{G}_{m} \rightarrow 1
$$

the $\ell$-torsion of $\mathrm{Br}^{\prime}(\mathcal{X})$ is the image of $\mathrm{H}_{\text {lis-ét }}^{2}\left(\mathcal{X}, \mu_{\ell}\right)$. Now, given elements

$$
\alpha \in \mathrm{H}_{\text {lis-ét }}^{1}\left(\mathcal{X}, \mu_{\ell}\right), \quad \beta \in \mathrm{H}_{\text {lis-êt }}^{1}(\mathcal{X}, \mathbb{Z} / \ell \mathbb{Z}),
$$

there exists a canonical Azumaya algebra $\mathcal{A}_{\alpha, \beta}$ whose class in $\mathrm{H}_{\text {lis-êt }}^{2}\left(\mathcal{X}, \mu_{\ell}\right)$ is equal to the cup product

$$
\alpha \cdot \beta \in \mathrm{H}_{\text {lis-ét }}^{2}\left(\mathcal{X}, \mu_{\ell}\right)=\operatorname{Br}^{\prime}(\mathcal{X})_{\ell} .
$$

In particular, any element of $\mathrm{Br}^{\prime}$ that can be written in this way automatically belongs to the Brauer group.

The equality $\operatorname{Br}(\mathcal{X})=\operatorname{Br}^{\prime}(\mathcal{X})$, at least for the prime-to-char $(k)$ part, is known to hold for a large class of Deligne-Mumford stacks: the following theorem is a combination of a result by Edidin, Hassett, Kresch and Vistoli [11, Thm. 3.6] ${ }^{1}$, which says that an element $\alpha \in \operatorname{Br}^{\prime}(\mathcal{X})$ comes from the Brauer group if and only if the corresponding $\mu_{n}$-gerbe is a quotient stack, and a result by Kresch and Vistoli [18, Thm. 2.2], which gives a criterion for a Deligne-Mumford stack to be a quotient stack.

Theorem 2.1 (EHKV). Let $\mathcal{X}$ be a smooth, separated, generically tame Deligne-Mumford stack of finite type over $k$, and assume that $\mathcal{X}$ has a quasi-projective coarse moduli space. Then we have ${ }^{c} \operatorname{Br}(\mathcal{X})={ }^{c} \mathrm{Br}^{\prime}(\mathcal{X})$.

In particular, the hypotheses of this theorem are valid for the stacks $\mathcal{H}_{g}$ of hyperelliptic curves and for the stack $\mathcal{M}_{1,1}$ of elliptic curves, so we will know a priori that all the elements we produce belong to the Brauer group. We remark that the explicit descriptions of the elements we give would also be sufficient to show that they all belong to the Brauer group.

Finally, the following ensures that the equality $\operatorname{Br}(\mathcal{X})=\operatorname{Br}^{\prime}(\mathcal{X})$ holds for all smooth finite DeligneMumford stacks, with no tameness requirement:

Proposition 2.2. Let $\mathcal{X}$ be a Deligne-Mumford stack, finite and smooth over $k$. Then $\operatorname{Br}(\mathcal{X})=\operatorname{Br}^{\prime}(\mathcal{X})$.

Proof. By [14, Ch.II, Lemma 4], given a surjective, finite and locally free map $Y \stackrel{f}{\rightarrow} X$, if $\alpha \in \operatorname{Br}^{\prime}(X)$ and $f^{*} \alpha \in \operatorname{Br}(Y)$, then $\alpha \in \operatorname{Br}(X)$. The result is proven in the setting of strictly ringed topoi, so in particular it holds for Deligne-Mumford stacks.

Then we can just apply this to $X \rightarrow \mathcal{X}$, where $X$ is an affine scheme and the map $X \rightarrow \mathcal{X}$ is an étale covering, and use the fact that for zero-dimensional schemes it is always true that $\mathrm{Br}=\mathrm{Br}^{\prime}$.

${ }^{1}$ The theorem is stated for Noetherian schemes, but the key lemmas needed for the proof, in Section 2, are all formulated in terms of Deligne-Mumford stacks, and the proof of the theorem carries word by word for a separated Noetherian Deligne-Mumford stack. 


\subsection{Generalised cohomological invariants}

Classically, cohomological invariants are defined as natural transformations from the functor

$$
\mathrm{T}_{G}:(\text { Field } / k) \rightarrow(\text { Set }), \quad \mathrm{T}_{G}(F) \stackrel{\text { def }}{=}\{G \text {-torsors over } F / \text { iso }\},
$$

which sends a field $F$ to the isomorphism classes of $G$-torsors over $F$, to the twisted cohomology functor

$$
\mathrm{H}_{D}:(\text { Field } / k) \rightarrow(\text { Set }), \quad \mathrm{H}_{D}^{\bullet}(F) \stackrel{\text { def }}{=} \oplus_{i} \mathrm{H}_{\mathrm{Gal}}^{i}(F, D(i)),
$$

where $D$ is a torsion Galois module. This is the definition in Garibaldi, Merkurjev and Serre's book [15].

In [22], the second author extended the notion of cohomological invariants to a theory of invariants for algebraic stacks, retrieving the classical theory when the stack is equal to the classying stack of $G$-torsors $\mathrm{B} G$.

In both the classical case and the more general version of cohomological invariants that we use in this paper, one can take a more general approach regarding coefficients and define cohomological invariants as natural transformations to any of Rost's cyle modules, as defined in [24].

These kind of generalised invariants were considered by both Guillot [17, Sec. 6] and the second author [22, Sec. 6], but as recently pointed out by Gille and Hirsch [16, Sec. 3], both approaches work only for cycle modules defined over every extension of the base field $k$, such as Galois cohomology and Milnor's K-theory, while in general a cycle module M is defined only over finitely generated extensions of $k$.

In their paper, Gille and Hirsch rework the foundations of the theory in this more general case, and prove a splitting formula for finite reflection groups. Following their approach, in this section we give a corrected definition of generalised cohomological invariants of algebraic stacks and show that the usual properties apply. In Section 5, we will use equivariant techniques to obtain a sharpening of their splitting formula.

Let us recall the construction of Rost's cycle modules [24]. Given a field $F$, denote by $\mathrm{T}\left(F^{*}\right)$ the tensor ring $\oplus_{i \geq 0}\left(F^{*}\right)^{\otimes_{\mathbb{Z}} i}$. Milnor's K-theory ring $\mathrm{K}_{\mathrm{Mil}}^{\bullet}(F)$ is given by

$$
\mathrm{K}_{\mathrm{Mil}}^{\bullet}(F)=\mathrm{T}\left(F^{*}\right) /\left\{a \otimes b \mid a, b \in F^{*}, a+b=1\right\} .
$$

For a field extension $\phi: F \rightarrow F^{\prime}$ there is a map $\phi^{*}: \mathrm{K}_{\mathrm{Mil}}^{\bullet}(F) \rightarrow \mathrm{K}_{\text {Mil }}^{\bullet}\left(F^{\prime}\right)$ given by restriction of scalars. If the extension is finite, we have a map $\phi_{*}: \mathrm{K}_{\text {Mil }}^{\bullet}\left(F^{\prime}\right) \rightarrow \mathrm{K}_{\text {Mil }}^{\bullet}(F)$ given by the norm map. These maps are functorial and compatible with each other. Moreover, we have a projection formula

$$
\phi_{*} \circ \phi^{*}=\left[F^{\prime}: F\right] \operatorname{Id}_{\mathrm{K}_{\mathrm{Mil}}^{*}}(F) .
$$

If the extension $F^{\prime} / F$ is purely inseparable, we moreover have the opposite equality,

$$
\phi^{*} \circ \phi_{*}=\left[F^{\prime}: F\right] \operatorname{Id}_{\mathrm{K}_{\mathrm{Mil}}\left(F^{\prime}\right)} \cdot
$$

We call a discreet valuation ring (DVR) $(R, v)$ geometric if $R$ is a $k$-algebra and the transcendence degree of the quotient field $F_{R}$ is 1 higher than the transcendence degree of the residue field $F_{v}$. In this case we have a boundary map

$$
\partial_{v}: \mathrm{K}_{\mathrm{Mil}}^{\bullet}\left(F_{R}\right) \rightarrow \mathrm{K}_{\mathrm{Mil}}^{\bullet-1}\left(F_{v}\right)
$$

In degree 1 , this map is just the valuation $v$. Finally, let $(R, v)$ be as before and let $\pi$ be a uniformiser for v. We define a map

$$
s_{v}^{\pi}: \mathrm{K}_{\mathrm{Mil}}^{\bullet}\left(F_{R}\right) \rightarrow \mathrm{K}_{\mathrm{Mil}}^{\bullet}\left(F_{v}\right), \quad s_{v}^{\pi}(x)=\partial_{v}(\pi \cdot x) .
$$

If $\partial_{v}(x)=0$, the map does not depend on the choice of $\pi$ and we just denote it $s_{v}$. 
Milnor's K-theory is the basic, and most important, cycle module. In general, denote by $\mathfrak{F}_{k}$ the subcategory of (Field/ $k$ ) given by finitely generated extensions. A cycle module $\mathrm{M}$ is a contravariant functor ${ }^{2}$ from $\mathfrak{F}_{k}^{\text {op }}$ to the category of graded abelian groups, equipped with four operations $\phi_{*}, \phi^{*}, \partial_{v}, s_{v}^{\pi}$. This collection of data has to satisfy a long list of properties, which in particular imply that $\mathrm{M}^{\bullet}(F)$ has to be a $\mathrm{K}_{\mathrm{M}}^{\bullet}(F)$-module for all $F$, the operations have to be compatible with those on $\mathrm{K}_{\mathrm{Mil}}$ and with each other and they have to satisfy the projection formula already given [24, Sec. 1-2].

The key examples of cycle modules other than Milnor's K-theory are given by the twisted Galois cohomology cycle modules $H_{D}$. Note that by Voevodsky's norm-residue isomorphism [29, Thm. 6.1] or by the Merkurjev-Suslin theorem in degree 2 or lower, we have the equality

$$
\mathrm{K}_{\mathrm{Mil}} /(\ell)=\mathrm{H}_{\mathbb{Z} / \ell \mathbb{Z}} \stackrel{\text { def }}{=} \mathrm{K}_{\ell} .
$$

Given a geometric DVR $(R, v)$, Rost defines a group $\mathrm{M}^{\bullet}(v)$ (not to be confused with $\mathrm{M}^{\bullet}\left(F_{v}\right)$ ) with a map $p: \mathbf{M}^{\bullet}\left(F_{R}\right) \rightarrow \mathbf{M}^{\bullet}(v)$. The group sits in the exact sequence

$$
0 \rightarrow \mathrm{M}^{\bullet}\left(F_{v}\right) \stackrel{i}{\rightarrow} \mathrm{M}^{\bullet}(v) \stackrel{\partial}{\rightarrow} \mathrm{M}^{\bullet-1}\left(F_{v}\right) \rightarrow 0,
$$

and the composition $\partial \circ p$ is equal to $\partial_{v}$.

When working with Galois cohomology, the group $\mathrm{H}_{D}^{\bullet}(v)$ is equal to $\mathrm{H}_{D}^{\bullet}\left(F_{R^{h}}\right)$, where $\left(R^{h}, v\right)$ is the Henselisation of $(R, v)$ [15, Eq. (7.10)]. The definition of cohomological invariants of an algebraic stack [22, Def. 2.2] includes a continuity condition which can be rephrased as stating that for a Henselian DVR $R^{h}$, the value of $p\left(\alpha\left(F_{R^{h}}\right)\right)$ should be equal to $i\left(\alpha\left(F_{v}\right)\right)$. This suggests the following general definition:

Definition 2.3. Let $\mathcal{X}$ be an algebraic stack. A cohomological invariant with coefficients in $\mathrm{M}$ is a natural transformation

$$
\alpha: \mathrm{Pt}_{\mathcal{X}} \longrightarrow \mathrm{M}
$$

such that for any geometric $\operatorname{DVR}(R, v)$ and any map from the spectrum of $R$ to $\mathcal{X}$ we have

$$
p\left(\alpha\left(F_{R}\right)\right)=i\left(\alpha\left(F_{v}\right)\right) \in \mathbf{M}^{\bullet}(v) .
$$

The cohomological invariants with coefficients in $\mathrm{M}$ of $\mathcal{X}$ form a graded $\mathrm{M}^{\bullet}(k)$-module $\operatorname{Inv}^{\bullet}(\mathcal{X}, \mathrm{M})$.

Given a morphism $f: \mathcal{Y} \rightarrow \mathcal{X}$, we define a pullback $f^{*}: \operatorname{Inv}^{\bullet}(\mathcal{X}, \mathbf{M}) \rightarrow \operatorname{Inv}^{\bullet}(\mathcal{Y}, \mathbf{M})$ by setting $f^{*}(\alpha(q))=\alpha(f(q))$.

Throughout the paper, every decomposition of a group of cohomological invariants will be a decomposition of $\mathbf{M}^{\bullet}(k)$-modules, unless specified otherwise.

Note that this definition differs from the one in [22, Sec. 6], as it requires the continuity condition on geometric DVRs rather than Henselian ones. This has the advantage that it applies to more general cycle modules, as in general a cycle module is only defined on finitely generated extensions of the base field. As will be evident in the rest of the section, the theory we obtain with this definition is exactly the same as what we obtained in [22].

Definition 2.4. The group $\operatorname{Inv}^{\bullet}(\operatorname{Spec}(k), \mathrm{M})$ is equal to $\mathrm{M}^{\bullet}(k)$. Consider a stack $\mathcal{X} \stackrel{\pi}{\rightarrow} \operatorname{Spec}(k)$. Its invariants coming from the pullback of $\operatorname{Inv}{ }^{\bullet}(\operatorname{Spec}(k), \mathbf{M})=\mathbf{M}^{\bullet}(k)$ are called constant. The value of these invariants at a point $q: \operatorname{Spec}(F) \rightarrow \mathcal{X}$ depends only on the field $F$.

The group of normalised cohomological invariants of $\mathcal{X}$ is

$$
\widetilde{\operatorname{Inv}^{\bullet}}(\mathcal{X}, \mathbf{M})=\operatorname{Inv}^{\bullet}(\mathcal{X}) / \pi^{*} \operatorname{Inv}{ }^{\bullet}(k, \mathbf{M})
$$

\footnotetext{
${ }^{2}$ We choose to see cycle modules as contravariant functors from the opposite category, contrary to what Rost does, so that the pullback/push-forward notation agrees with that for Chow groups with coefficients.
} 
If $\mathcal{X}=\mathrm{B} G$, the group of normalised invariants is equal to the $\operatorname{subgroup}$ of $\operatorname{Inv}^{\bullet}(\mathrm{B} G, M)$ given by invariants which are zero on the trivial torsor.

Remark 2.5. In our notation, we call $\operatorname{Inv}^{i}(\mathrm{~B} G, \mathrm{M})$ the group denoted by $\operatorname{Inv}^{i}(G, \mathrm{M})$ in [16], and $\operatorname{Inv}^{i}\left(\mathrm{~B} G, \mathrm{H}_{D}\right)$ the group denoted by $\operatorname{Inv}^{i}(G, D)$ in [15].

Remark 2.6. Given an étale extension $\left(R^{\prime}, v^{\prime}\right)$ of $(R, v)$ with the same residue field $F_{v}=F=F_{v^{\prime}}$, we have an isomorphism $\mathbf{M}^{\bullet}(v) \simeq \mathrm{M}^{\bullet}\left(v^{\prime}\right)$ and the continuity condition for $\left(R^{\prime}, v^{\prime}\right)$ is equivalent to the continuity condition for $(R, v)$.

In other words, we can check the continuity condition on any Nisnevich neighbourhood of the closed point of $\operatorname{Spec}(R)$.

Moreover, if $M$ is defined on all extensions of $k$, we can restrict to checking the condition on the Henselisation $R^{h}$.

Recall that a smooth Nisnevich morphism [22, Def. 3.2] is a representable smooth morphism $f$ : $\mathcal{Y} \rightarrow \mathcal{X}$ of algebraic stacks such that for any field extension $F / k$ and any point $p: \operatorname{Spec}(F) \rightarrow \mathcal{X}$, we have a lifting

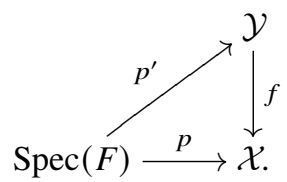

A smooth $\ell$-Nisnevich morphism [22, Def. 3.4] is a representable smooth morphism $f: \mathcal{Y} \rightarrow \mathcal{X}$ of algebraic stacks such that for any point $p: \operatorname{Spec}(F) \rightarrow \mathcal{X}$, we have a commutative square

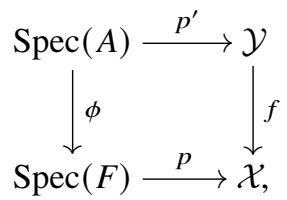

where $A=F_{1} \times \cdots \times F_{r}$, the extension $F_{i} / F$ is finite and separable for each $i$ and $\left(\left[F_{1}: F\right], \ldots,\left[F_{r}: F\right], \ell\right)=1$.

Given an algebraic stack $\mathcal{X}$ we define the smooth Nisevich and smooth $\ell$-Nisnevich sites of $\mathcal{X}$ as the sites $(\mathrm{Spc} / \mathcal{X})_{\mathrm{sm}-\mathrm{Nis}}$ and $(\mathrm{Spc} / \mathcal{X})_{\mathrm{sm} \ell \text {-Nis }}$ where the objects are representable maps to $\mathcal{X}$, morphisms are commutative squares over the identity of $\mathcal{X}$ and coverings are respectively smooth Nisnevich and smooth $\ell$-Nisnevich morphisms [22, Def. 3.9].

Let $A$ be as before. Then the fibred product $\operatorname{Spec}(A) \times_{\operatorname{Spec}(F)} \operatorname{Spec}(A)$ is a disjoint union $\operatorname{Spec}\left(E_{1}\right) \sqcup$ $\cdots \sqcup \operatorname{Spec}\left(E_{n}\right)$, where each of the $E_{j}$ is a finite separable extension of $F$. There are two pullbacks $\operatorname{Pr}_{1}^{*}, \operatorname{Pr}_{2}^{*}: \oplus_{i} M\left(F_{i}\right) \rightarrow \oplus_{j} \mathrm{M}\left(E_{j}\right)$. We have a sequence

$$
0 \rightarrow \mathrm{M}(F) \rightarrow \oplus_{i} \mathrm{M}\left(F_{i}\right) \stackrel{\mathrm{Pr}_{1}^{*}-\mathrm{Pr}_{2}^{*}}{\longrightarrow} \oplus_{j} \mathrm{M}\left(E_{j}\right)
$$

which is always left exact when $\mathrm{M}$ is $\ell$-torsion. We say that $\mathrm{M}$ has the property $\left(\mathcal{S}_{\ell}\right)$ if $\mathrm{M}$ is $\ell$-torsion and the foregoing sequence is exact for any $F \in \mathfrak{F}_{k}$ and $A$ as before. This is true in particular for $\mathrm{M}=\mathrm{H}_{D}$, where $D$ is an $\ell$-torsion Galois module [22, Lm. 3.7].

Theorem 2.7. The functor $\operatorname{Inv}^{\bullet}(-, M)$ is a smooth Nisnevich sheaf.

If $\mathrm{M}$ is $\ell$-torsion, then the pullback of $\operatorname{Inv}^{\bullet}(-, \mathrm{M})$ through a smooth $\ell$-Nisnevich covering is injective.

If, moreover, $\mathrm{M}$ has the property $\left(\mathcal{S}_{\ell}\right)$, then $\operatorname{Inv}^{\bullet}(-, \mathrm{M})$ is a smooth $\ell$-Nisnevich sheaf.

Proof. The proof is very similar to the proof of [22, Thm. 3.8]. Let $\pi: \mathcal{Y} \rightarrow \mathcal{X}$ be a smooth Nisnevich covering. We define an invariant $\alpha$ by setting $\alpha(p)=\alpha\left(p^{\prime}\right)$ for any lifting $p^{\prime}$ of $p$. This is clearly well defined and functorial, thanks to the sheaf condition. 
Now let $(R, v)$ be a geometric DVR and let $f: \operatorname{Spec}(R) \rightarrow \mathcal{X}$ be a morphism. Let $R^{h}$ be the Henselisation of $R$. The morphism $\mathcal{Y} \times{ }_{\mathcal{X}} \operatorname{Spec}\left(R^{h}\right) \rightarrow \operatorname{Spec}\left(R^{h}\right)$ always has a section. This implies that we can find some finite extension $\left(R^{\prime}, v^{\prime}\right)$ of $R$ with the same residue field $F_{v}$ and a commutative square

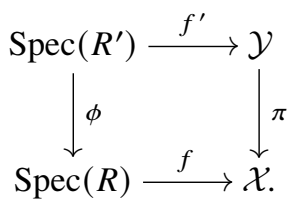

The continuity condition for $\left(R^{\prime}, v^{\prime}\right)$ implies the continuity condition for $(R, v)$, so we have proven our claim in the smoothNisnevich case.

Injectivity in the smooth $\ell$-Nisnevich case is trivial, because given an extension $F \subset F_{1} \times \cdots \times F_{r}$ as before, the map

$$
\phi_{1}^{*} \times \cdots \times \phi_{r}^{*}: \mathrm{M}^{\bullet}(F) \rightarrow \mathrm{M}^{\bullet}\left(F_{1}\right) \oplus \cdots \oplus \mathrm{M}^{\bullet}\left(F_{r}\right)
$$

is injective. If condition $\left(\mathcal{S}_{\ell}\right)$ holds, then we can proceed exactly as before to show that cohomological invariants form a smooth $\ell$-Nisnevich sheaf.

Remark 2.8. The typical example of smooth Nisnevich covering that will appear in the following is a quotient $X \rightarrow[X / G]$, where $G$ is a smooth and affine algebraic group over $k$ that is special in the sense that every $G$-torsor is Zariski locally trivial. In this case, the inverse image of a point $\operatorname{Spec}(F) \rightarrow[X / G]$ is a $G$-torsor over $\operatorname{Spec}(F)$, which is necessarily trivial and thus has a point, showing that $X \rightarrow[X / G]$ is smooth Nisnevich.

As a direct consequence of the continuity condition, we see that given a geometric DVR $R$, the value of a cohomological invariant being 0 at the quotient field implies the same for the residue field. Then it is easy to prove by induction that this property propagates to regular local rings and regular schemes of finite type.

Lemma 2.9. Let $X$ be a regular, connected scheme of finite type over $k$, with generic point $\xi$. Then for any $\alpha \in \operatorname{Inv}^{\bullet}(X, \mathrm{M})$, we have

$$
\alpha(\xi)=0 \Rightarrow \alpha=0 .
$$

Proof. This is proven exactly as in [16, Cor. 3.6] or [22, Lm. 4.5].

Given a smooth, irreducible scheme $X$ over $k$, the 0-codimensional Chow group with coefficients $A^{0}(X, \mathrm{M})$ is the subgroup of $\mathrm{M}(k(X))$ of elements such that $\partial_{v} a=0$ whenever $(R, v)$ is the local ring of a point $x \in X^{(1)}-$ that is, a point of codimension 1 in $X$.

The following theorem shows that on smooth schemes, this group is equal to the group of cohomological invariants, and consequently in general the functor of cohomological invariants is a sheafification of $A^{0}(-, \mathrm{M})$ in the smooth Nisnevich (or smooth $\ell$-Nisnevich) topology. The properties of Chow groups with coefficients will be discussed in depth in Subsection 2.4.

Theorem 2.10. Let $X$ be a scheme, smooth over $k$. Then

$$
\operatorname{Inv}^{\bullet}(X, \mathrm{M})=A^{0}(X, \mathrm{M})
$$

Let $\mathcal{X}$ be an algebraic stack, smooth over $k$. Then the functor $\operatorname{Inv}^{\bullet}(-, \mathrm{M})$ is the smooth Nisnevich sheafification of $A^{0}(-, \mathrm{M})$.

Proof. The second statement is an obvious consequence of the first and Theorem 2.7.

To prove the first statement, let $X$ be a smooth, connected scheme over $k$ with generic point $\xi$. Note that given an invariant $\alpha$, the value $\alpha(\xi)$ is unramified at all points $x \in X^{(1)}$, so $\alpha(\xi) \in A^{0}(X$, M). This map 
is injective by the Lemma 2.9. On the other hand, an element $\beta \in A^{0}(X, \mathrm{M})$ defines a cohomological invariant by pullback (see [24, p. 360, after Cor. 6.5]), and the two maps are inverse to each other.

Remark 2.11. When $\mathrm{M}=\mathrm{H}_{D}$, by the Bloch-Ogus-Gabber exact sequence we have

$$
\operatorname{Inv}^{\bullet}\left(X, \mathrm{H}_{D}\right)=A^{0}\left(X, \mathrm{H}_{D}\right)=\mathrm{H}_{\mathrm{Zar}}^{0}\left(X, \mathrm{H}^{\bullet}(-, D)\right) .
$$

That is, on smooth schemes, cohomological invariants are the Zariski sheafification of the twisted cohomology functor $\mathrm{H}^{\bullet}(-, D)$.

Corollary 2.12. Let $f: \mathcal{Y} \rightarrow \mathcal{X}$ be a map of smooth algebraic stacks over $k$. If $f$ is either

$\circ$ an affine bundle or

- an open immersion whose complement has codimension at least 2,

then the pullback $f^{*}$ is an isomorphism.

Proof. This is an easy consequence of the same properties being true for $A^{0}(X, \mathrm{M})([24, \mathrm{Sec} .9]$ for the first statement, and the second one by definition) and the sheaf conditions.

Finally, the following proposition shows that when working with an $\ell$-torsion cycle module, we can always reduce to $\ell$ being a prime power:

Proposition 2.13. Assume that $\ell=r s$, with $(r, s)=1$, and let $\mathrm{M}$ be an $\ell$-torsion cycle module. Then the following hold:

1. $\mathrm{M}_{r}$ and $\mathrm{M}_{s}$ are both cycle modules.

2. We have $\mathrm{M}=\mathrm{M}_{r} \times \mathrm{M}_{s}$, and if $\mathrm{M}$ has a product pairing, the product respects the ring structure.

3. We have $\operatorname{Inv}^{\bullet}(\mathcal{X}, \mathrm{M})=\operatorname{Inv}^{\bullet}\left(\mathcal{X}, \mathrm{M}_{r}\right) \oplus \operatorname{Inv}^{\bullet}\left(\mathcal{X}, \mathrm{M}_{s}\right)$.

Proof. The four operations $\phi^{*}, \phi_{*}, \partial_{v}, s_{v}^{\pi}$ all send $\mathrm{M}_{r}$ to itself. Verifying that the requirements in [24, Sec. 1-2] are all satisfied is a routine exercise, proving (1). Moreover, we have maps $\mathrm{M} \stackrel{\cdot s}{\rightarrow} \mathbf{M}_{r}, \mathrm{M} \stackrel{\cdot r}{\rightarrow} \mathbf{M}_{s}$. The resulting map $\mathrm{M} \stackrel{\rho}{\rightarrow} \mathrm{M}_{r} \times \mathrm{M}_{s}$ is clearly an isomorphism, proving (2). Finally, the map $\rho$ induces a map at the level of cohomological invariants, which again is easily shown to be an isomorphism, proving (3).

\subsection{Cohomological invariants and the cohomological Brauer group}

In this subsection we restate some results from the last section of [22] connecting cohomological invariants and the cohomological Brauer group, and we extend them to quotient stacks. Recall that $\ell$ is a positive number not divisible by $\operatorname{char}(k)$.

Lemma 2.14. Let $X$ be an algebraic space, quasi-separated and smooth over $k$. Then

$$
\operatorname{Inv}^{2}\left(X, \mathrm{H}_{\mu_{\ell}}\right)=\operatorname{Br}^{\prime}(X)_{\ell} .
$$

Proof. This is the content of [22, Lem. 7.6].

The proof of this statement does not extend to algebraic stacks, but as for cohomological invariants, any theory that is invariant by the operations of removing closed subsets of high codimension and of passing to vector bundles admits an extension to quotient stacks through standard equivariant techniques. The following lemma tells us exactly that:

Lemma 2.15. Let $\mathcal{X}$ be an algebraic stack smooth over $k$. Then the following are true:

- If $\mathcal{V} \rightarrow \mathcal{X}$ is a vector bundle, we have $\operatorname{Br}^{\prime}(\mathcal{X})_{\ell}=\operatorname{Br}^{\prime}(\mathcal{V})_{\ell}$.

- If $\mathcal{U} \subset \mathcal{X}$ is an open subset whose complement has codimension $\geq 2$, we have $\operatorname{Br}^{\prime}(\mathcal{X})_{\ell}=\operatorname{Br}^{\prime}(\mathcal{U})_{\ell}$. 
Proof. This is proven in [12, Prop. 1.3, 1.4].

Now we can use equivariant approximation to reduce the problem of computing the cohomological Brauer group to algebraic spaces. The following ideas will be vastly expanded on in Section 4 .

Lemma 2.16. Let $G / k$ be an affine, smooth algebraic group, and $i$ a positive integer. Then there exists a representation $V$ with an open subset $U$ such that $G$ acts freely on $U$ and the codimension of the complement of $U$ is at least $i$.

Proof. We embed $G$ into $\mathrm{GL}_{n}$, which can always be done for a smooth affine algebraic group over $k$. Now consider the action of $\mathrm{GL}_{n}$ on $n \times n+i$ matrices through left multiplication. The action is free on the open subset of matrices of maximal rank, whose complement has codimension $i$. This is contained in [10, Lemma 9].

Proposition 2.17. Let $X$ be an algebraic space, quasi-separated and smooth over $k$, being acted upon by an affine smooth group scheme $G / k$. Then

$$
\operatorname{Inv}^{2}\left([X / G], \mathrm{H}_{\mu_{\ell}^{v}}\right)=\operatorname{Br}^{\prime}([X / G])_{\ell} .
$$

Proof. Let $U$ be as in Lemma 2.16, with $i=2$. Then $(U \times X) / G$ is a smooth algebraic space, as the action is free. As the map $(U \times X) / G \rightarrow[X / G]$ is the composition of a vector bundle and an open immersion whose complement has codimension 2 or higher, it has the same cohomological Brauer group and cohomological invariants as $[X / G]$. Lemma 2.14 allows us to conclude.

Finally, the following lemmas will be useful in detecting cyclic algebras among elements of the Brauer groups.

Lemma 2.18. Let $X / k$ be a smooth and separated algebraic space over $k$, being acted upon properly by an affine smooth group scheme $G / k$. Then

$$
\operatorname{Inv}^{1}\left([X / G], \mathrm{K}_{\ell}\right)=\mathrm{H}^{1}\left([X / G], \mu_{\ell}\right) .
$$

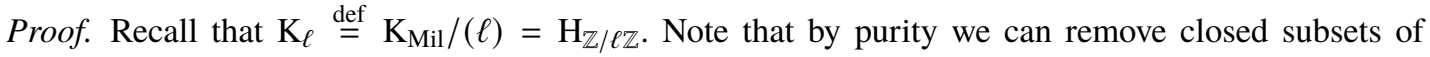
codimension $\geq 2$ without affecting the first cohomology groups. By the process already described, it is then sufficient to prove the statement for a smooth separated algebraic space $X$.

Moreover, by [27, Tag 0ADD] we know that up to a closed subset of codimension at most $2, X$ is a scheme, so we can reduce to a smooth scheme $X$.

Consider the morphism of sites

$$
\left(i_{*}, i^{*}\right): X_{\text {ét }} \rightarrow X_{\mathrm{Zar}}
$$

given by restriction and pullback. The composition with the global sections functor $\Gamma$ induces a Grothendieck spectral sequence

$$
\mathrm{H}_{\mathrm{Zar}}^{p}\left(X, \mathrm{R}^{q} i_{*} F\right) \Rightarrow \mathrm{H}_{\mathrm{et}}^{p+q}(X, F) .
$$

Picking $F=\mu_{\ell}$, the low-degree-terms exact sequence reads

$$
0 \rightarrow \mathrm{H}_{\mathrm{Zar}}^{1}\left(X, \mu_{\ell}\right) \rightarrow \mathrm{H}_{\mathrm{et}}^{1}\left(X, \mu_{\ell}\right) \rightarrow \operatorname{Inv}^{1}\left(X, \mathrm{~K}_{\ell}\right) \rightarrow \mathrm{H}_{\mathrm{Zar}}^{2}\left(X, \mu_{\ell}\right) .
$$

Now observe that on the small Zariski site of $X$, the sheaf $\mu_{\ell}$ is constant, and thus flasque. Then its Zariski cohomology is trivial and the result follows immediately.

Lemma 2.19. Let $\mathcal{X}$ be an algebraic stack over $k$. Any element of $\operatorname{Br}^{\prime}(\mathcal{X})$ coming from the cup product $\mathrm{H}^{1}\left(\mathcal{X}, \mu_{\ell}\right) \otimes \mathrm{H}^{1}(\mathcal{X}, \mathbb{Z} / \ell \mathbb{Z}) \rightarrow \mathrm{H}^{2}\left(\mathcal{X}, \mu_{\ell}\right)$ is represented by a cyclic algebra.

Proof. This is proven in $[1,2.10,3.7]$. 


\subsection{Chow groups with coefficients}

As our main tool for computations, we need to establish the main properties of the theory of Chow groups with coefficients. Rost's original paper [24] develops the theory for arbitrary cycle modules, but it notably lacks a theory of Chern classes, which was later developed in [21, 2.3-2.5].

A cycle with coefficients $\alpha \in C_{i}(X, \mathrm{M})$ is a formal finite sum of elements in the form $(V, \tau)$, where $V$ is an irreducible subscheme of $X$ of dimension $i$ and $\tau \in \mathbf{M}^{\bullet}(k(V))$. The groups $C_{i}(X, \mathbf{M})$ form a complex

$$
0 \rightarrow C_{\operatorname{dim}(X)}(X, \mathrm{M}) \rightarrow \cdots \rightarrow C_{1}(X, \mathrm{M}) \rightarrow C_{0}(X, \mathrm{M}) \rightarrow 0 .
$$

If $V$ is a normal irreducible subscheme, the differential $\mathrm{d}$ is defined on an element $(V, \tau)$ by

$$
\mathrm{d}(V, \tau)=\oplus_{p \in V^{(1)}}\left(\bar{p}, \partial_{v_{p}} \tau\right)
$$

where the sum runs over points of codimension 1 and the valuation $v_{p}$ is defined by the DVR $\mathcal{O}_{V, p}$. There is some extra subtlety involved in the general definition when the subscheme $V$ is not assumed to be normal.

The axioms of cycle modules ensure that the sum is finite and that $\mathrm{d} \circ \mathrm{d}=0$. The groups $A_{i}(X, \mathrm{M})$ are defined as the homology of this complex.

When $X$ is equidimensional, which will always be the case in the following, we can define $C^{i}(X, \mathrm{M})$ as the group of cycles of codimension $i$, and switch to the codimension notation

$$
A^{i}(X, \mathrm{M})=A_{\operatorname{dim}(X)-i}(X, \mathrm{M})
$$

which is better suited for our purposes. We denote

$$
A^{*}(X, \mathbf{M})=\bigoplus_{i=0}^{\operatorname{dim}(X)} A^{i}(X, \mathbf{M})
$$

The group $A^{*}(X, \mathrm{M})$ has two different gradations, one given by codimension and one by the gradation on $\mathrm{M}$. We will always refer to the former by codimension and the latter by degree.

We recall the main properties we are interested in:

Proposition 2.20. Let $X$ and $Y$ be equidimensional, quasi-projective schemes of finite type over $k$. Let $\mathrm{M}$ and $\mathrm{N}$ be $\ell$-torsion cycle modules. Then we have the following properties:

1. Proper push-forward: every proper morphism $f: X \rightarrow Y$ induces a homomorphism of groups

$$
f_{*}: A_{i}(X, \mathrm{M}) \longrightarrow A_{i}(Y, \mathrm{M})
$$

which preserves the cohomological degree.

2. Flat pullback: every flat morphism $f: X \rightarrow Y$ of relative constant dimension induces a homomorphism of groups

$$
f^{*}: A^{i}(Y, \mathrm{M}) \longrightarrow A^{i}(X, \mathrm{M})
$$

which preserves the cohomological degree.

3. Localisation exact sequence: given a closed subscheme $Z \stackrel{i}{\hookrightarrow} X$ whose open complement is $U \stackrel{j}{\hookrightarrow} X$, there exists a long exact sequence

$$
\cdots \rightarrow A_{i}(X, \mathrm{M}) \stackrel{j^{*}}{\rightarrow} A_{i}(U, \mathrm{M}) \stackrel{\partial}{\rightarrow} A_{i-1}(Z, \mathrm{M}) \stackrel{i_{*}}{\rightarrow} A_{i-1}(X, \mathrm{M}) \rightarrow \cdots
$$


The boundary homomorphism $\partial$ has cohomological degree -1, whereas the other homomorphisms have cohomological degree 0.

4. Compatibility: given a Cartesian square of schemes

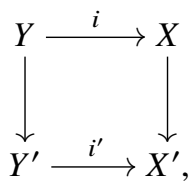

where the vertical morphisms are closed emebeddings and the horizontal ones are proper, we get a commutative square

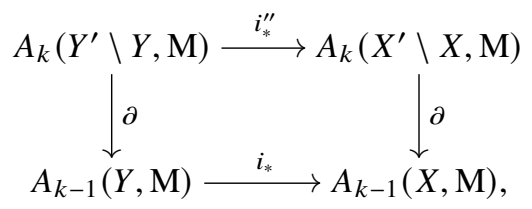

where $i^{\prime \prime}$ is the restriction of $i^{\prime}$ to $Y^{\prime} \backslash Y$.

5. Homotopy invariance: if $\pi: E \rightarrow X$ is a finite-rank vector bundle, then we have an isomorphism

$$
\pi^{*}: A^{i}(X, \mathrm{M}) \simeq A^{i}(E, \mathrm{M})
$$

which preserves the cohomological degrees.

6. Pullback along regular embeddings: every regular embedding $i: X \rightarrow Y$ induces a pullback morphism

$$
i^{*}: A^{i}(Y, \mathrm{M}) \longrightarrow A^{i}(X, \mathrm{M})
$$

which satisfies the usual functorial properties.

7. Pullback from smooth targets: every morphism $f: X \rightarrow Y$ with smooth target $Y$ induces a pullback morphism

$$
f^{*}: A^{i}(Y, \mathrm{M}) \longrightarrow A^{i}(X, \mathrm{M})
$$

which satisfies the usual functorial properties. Whenever $f$ is flat of relative constant dimension, this pullback coincides with the flat pullback introduced before.

8. Ring structure: if $X$ is smooth and $\mathrm{M}$ is a cycle module with a pairing, then $A^{*}(X, \mathrm{M})$ inherits the structure of a graded commutative ring, where the graded commutativity should be understood in the following sense: if $\alpha$ has codimension $i$ and degree $d$, and $\beta$ has codimension $j$ and degree $e$, then $\alpha \cdot \beta=(-1)^{\text {de }} \beta \cdot \alpha$, and the product has codimension $i+j$ and degree $d+e$.

9. Module structure: If $Y$ is smooth and we have pairings $\mathrm{N} \times \mathrm{N} \rightarrow \mathrm{N}$ and $\mathrm{N} \times \mathrm{M} \rightarrow \mathrm{M}$, then $A^{*}(Y, \mathrm{M})$ inherits the structure of an $A^{*}(Y, \mathrm{~N})$-module.

For every morphism $f: X \rightarrow Y$ and every $\alpha$ in $A^{*}(Y, \mathrm{~N})$ and $\beta$ in $A^{*}(X, \mathrm{M})$, we have

$$
f^{*}(\alpha \cdot \beta)=f^{*} \alpha \cdot f^{*} \beta
$$

For every proper morphism $f: X \rightarrow Y$, the following projection formula holds:

$$
f_{*}\left(\alpha \cdot f^{*} \beta\right)=f_{*} \alpha \cdot \beta \text {. }
$$


10. Chern classes: For a vector bundle $E \rightarrow X$ of finite rank $r$, we have well-defined Chern class homomorphisms

$$
c_{k}(E)(-): A^{i}(X, \mathrm{M}) \longrightarrow A^{i+k}(X, \mathrm{M})
$$

which satisfy the usual standard properties.

In particular, if $i: X \rightarrow E$ denotes the zero-section embedding, we have

$$
i^{*} i_{*}(\alpha)=c_{r}(E)(\alpha)
$$

11. Projective bundle formula: For a projective bundle $\pi: P(E) \rightarrow X$ whose fibres have dimension $r$, we have

$$
A^{i}(P(E), \mathrm{M}) \simeq \underset{j=0}{i} c_{1}(\mathcal{O}(1))^{j}\left(\pi^{*} A^{i-j}(X, \mathrm{M})\right)
$$

for $0 \leq i \leq r$. Moreover, we have

$$
c_{1}(\mathcal{O}(1))^{r+1}(-)=-\sum_{i=0}^{r} c_{1}(\mathcal{O}(1))^{i}\left(\pi^{*} c_{r+1-i}(E)(-)\right) .
$$

Sketch of Proof. The proofs of (1)-(4) are the content of [24, Sec. 4], (5) is [24, Prop. 8.6], (6) is [24, Prop. 12.3], (7) is [24, Thm. 12.1 and Prop. 12.2] and (8) and (9) are [24, Thm. 14.6]. The theory behind (10) and (11) is developed in [21, Sec. 2.1].

Proposition 2.21. Let $E$ be a vector bundle over a smooth variety $X$, and let $\mathrm{M}$ be an $\ell$-torsion cycle module. Let $\alpha$ be an element of $A^{*}(X, \mathrm{M})$.

The class $c_{i}(E)(\alpha) \in A^{*}(X, \mathrm{M})$ is equal to the image of $c_{i}(E)(1) \otimes \alpha$ through the multiplication map $A^{*}\left(X, \mathrm{H}_{\mathbb{Z} / \ell \mathbb{Z})} \otimes A^{*}(X, \mathrm{M}) \rightarrow A^{*}(X, \mathrm{M})\right.$.

Proof. Let $\pi: E \rightarrow X$ be a line bundle with zero section $i: X \rightarrow E$, so that $c_{1}(E)(\alpha)=i^{*} i_{*} \alpha$. We claim that

$$
i^{*} i_{*}(\alpha \cdot \beta)=i^{*} i_{*} \alpha \cdot \beta
$$

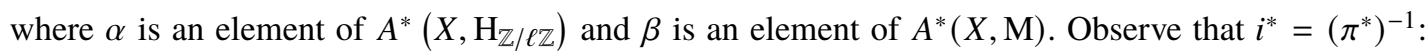
we will show that the pullback along $\pi$ of the two sides of equation (2.1) coincide. The pullback of the right-hand hand side is

$$
\pi^{*}\left(i^{*} i_{*} \alpha \cdot \beta\right)=\pi^{*} i^{*} i_{*} \alpha \cdot \pi^{*} \beta=i_{*} \alpha \cdot \pi^{*} \beta
$$

The pullback of the left-hand side is

$$
\pi^{*} i^{*} i_{*}(\alpha \cdot \beta)=i_{*}(\alpha \cdot \beta)=i_{*}\left(\alpha \cdot i^{*} \pi^{*} \beta\right)=i_{*} \alpha \cdot \pi^{*} \beta,
$$

where in the last equality we have used the projection formula (see Proposition 2.20(9)).

By taking $\alpha=1$ we obtain a proof of the proposition in the case of line bundles. The general case can be deduced from this one by applying the splitting principle and the Whitney summation formula.

Lemma 2.22. Let $X$ be a scheme and write $\mathbb{G}_{m}=\operatorname{Spec}\left(k\left[t, t^{-1}\right]\right)$. Let $\mathrm{M}$ be an $\ell$-torsion cycle module. Then

$$
A^{*}\left(X \times \mathbb{G}_{m}, \mathrm{M}\right)=A^{*}(X, \mathrm{M}) \oplus\{t\} A^{*}(X, \mathrm{M}),
$$

where $\{t\}$ is seen as an element of $\mathrm{H}^{1}\left(k(t), \mu_{\ell}\right) \simeq k(t)^{*} /\left(k(t)^{*}\right)^{\ell}$. 
Proof. Consider the localisation long exact sequence induced by the open embedding $X \times \mathbb{G}_{m} \subset X \times \mathbb{A}^{1}$, which is

$$
\cdots \rightarrow A^{i}\left(X \times \mathbb{A}^{1}, \mathrm{M}\right) \rightarrow A^{i}\left(X \times \mathbb{G}_{m}, \mathrm{M}\right) \rightarrow A^{i}(X \times\{0\}, \mathrm{M}) \rightarrow A^{i+1}\left(X \times \mathbb{A}^{1}, \mathrm{M}\right) \rightarrow \cdots
$$

We have $A^{i}\left(X \times \mathbb{A}^{1}, \mathrm{M}\right) \simeq A^{i}(X \times\{0\}, \mathrm{M}) \simeq A^{i}(X, \mathrm{M})$. With this identification, the last morphism in the sequence coincides with multiplication by $c_{1}\left(\mathcal{O}_{X}\right)=0$; hence for every $i$ we have

$$
0 \rightarrow A^{i}(X, \mathrm{M}) \rightarrow A^{i}\left(X \times \mathbb{G}_{m}, \mathrm{M}\right) \rightarrow A^{i}(X, \mathrm{M}) \rightarrow 0 .
$$

Let $t$ be the element in $\operatorname{Inv}^{\bullet}\left(X, \mathrm{H}_{\mathbb{Z} / \ell \mathbb{Z}}\right)$ which sends a morphism $\operatorname{Spec}(F) \rightarrow X \times \mathbb{G}_{m}$ to the equivalence class in $\mathrm{H}^{1}\left(F, \mu_{\ell}\right) \simeq F^{*} /\left(F^{*}\right)^{\ell}$ of the element in $F^{*}$ defined by $\operatorname{Spec}(F) \rightarrow X \times \mathbb{G}_{m} \rightarrow \mathbb{G}_{m}$.

We can use the invariant $t$, regarded as an element of $A^{0}\left(X \times \mathbb{G}_{m}, \mathrm{H}_{\mathbb{Z} / \ell \mathbb{Z}}\right)$ to define a morphism $i: A^{i}(X, \mathrm{M}) \rightarrow A^{i}\left(X \times \mathbb{G}_{m}, \mathrm{M}\right)$ by setting $i(\gamma)=t \cdot \operatorname{pr}_{1}^{*} \gamma$ (we are using here the fact that $A^{*}\left(X \times \mathbb{G}_{m}, \mathrm{M}\right)$ is an $A^{*}\left(X \times \mathbb{G}_{m}, \mathrm{H}_{\mathbb{Z} / \ell \mathbb{Z}}\right)$-module $)$.

It is easy to check that $i$ provides a splitting for the short exact sequence given, thus concluding the proof.

Proposition 2.23. Let $\mathrm{M}$ be an $\ell$-torsion cycle module. If $f: X \rightarrow Y$ is a universal homeomorphism, it induces an isomorphism $f_{*}: A^{*}(X, \mathrm{M}) \stackrel{\simeq}{\rightarrow} A^{*}(Y, \mathrm{M})$.

Proof. Let $f: X \rightarrow Y$ be a universal homeomorphism. Given a point $y \in Y$, its fibre $x$ is a point of $X$ and the map $f_{x}: x \rightarrow y$ is a purely inseparable field extension.

The two formulas

$$
\left(f_{x}\right)_{*}\left(\left(f_{x}\right)^{*} \alpha\right)=[k(x): k(y)] \alpha, \quad\left(f_{x}\right)^{*}\left(\left(f_{x}\right)_{*} \beta\right)=[k(x): k(y)] \beta,
$$

together with the fact that $\ell$ is not divisible by the characteristic of $k$, imply that both $\left(f_{x}\right)^{*}$ and $\left(f_{x}\right)_{*}$ are isomorphisms.

Then $f_{*}$ induces an isomorphism on cycle level, proving our claim.

\section{The Brauer group of $\mathcal{M}_{1,1}$}

The tools we developed up to this point are enough for a first demonstration of how our methods work.

The Brauer group of $\mathcal{M}_{1,1}$ has been explored in depth by Antieau and Meier in [1,20], including in mixed characteristic, which is beyond the reach of our tools. Our proof has some independent interest though, as it is much simpler than the techniques used in those papers.

Theorem 3.1. Assume that the characteristic of $k$ is different from 2 or 3 , and let $\mathrm{M}$ be an $\ell$-torsion cycle module. Then the cohomological invariants of $\mathcal{M}_{1,1}$ with coefficients in $\mathrm{M}$ are given by

$$
\operatorname{Inv}^{\bullet}\left(\mathcal{M}_{1,1}, \mathbf{M}\right) \simeq \mathbf{M}^{\bullet}(k) \oplus\left\{27 x^{2}+4 y^{3}\right\} \cdot \mathbf{M}^{\bullet}(k)_{12} .
$$

Proof. Consider the standard presentation of $\mathcal{M}_{1,1}$ as the quotient $\left[\left(\mathbb{A}^{2} \backslash V\right) / \mathbb{G}_{m}\right]$, where $V$ is the curve $27 x^{2}+4 y^{3}=0$ and $\mathbb{G}_{m}$ acts by $(x, y) \mapsto\left(x t^{6}, y t^{4}\right)$. The curve $V$ is universally homeomorphic to $\mathbb{A}^{1}$, so we have an exact sequence

$$
0 \rightarrow \mathrm{M}^{\bullet}(k) \rightarrow A^{0}\left(\mathbb{A}^{2} \backslash V, \mathrm{M}\right) \rightarrow \mathrm{M}^{\bullet}(k) \rightarrow 0 .
$$

The boundary of the element $\left\{27 x^{2}+4 y^{3}\right\} \in \mathrm{H}^{1}\left(k(x, y), \mu_{\ell}\right)$ at $V$ is 1 , and it is unramified every-

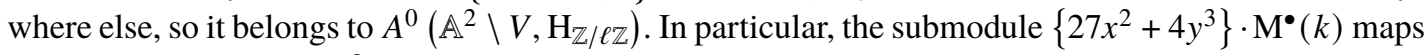
bijectively to $\mathbf{M}^{\bullet}(k)=A^{0}(V, \mathbf{M})$, splitting the exact sequence. Thus

$$
A^{0}\left(\mathbb{A}^{2} \backslash V, \mathbf{M}\right)=\mathbf{M}^{\bullet}(k) \oplus\left\{27 x^{2}+4 y^{3}\right\} \cdot \mathbf{M}^{\bullet}(k) .
$$


We have to understand which of these elements glue to invariants of $\mathcal{M}_{1,1}$. This is equivalent to checking whether the two pullbacks through

$$
\operatorname{pr}_{1}, \mathrm{~m}:\left(\mathbb{A}^{2} \backslash V\right) \times \mathbb{G}_{m}=\left(\mathbb{A}^{2} \backslash V\right) \times_{\mathcal{M}_{1,1}}\left(\mathbb{A}^{2} \backslash V\right) \rightrightarrows \mathbb{A}^{2} \backslash V
$$

coincide, where $\mathrm{m}$ denotes the multiplication map. By Lemma 2.22 we have

$$
A^{0}\left(\left(\mathbb{A}^{2} \backslash V\right) \times \mathbb{G}_{m}, \mathrm{M}\right)=A^{0}\left(\mathbb{A}^{2} \backslash V, \mathrm{M}\right) \oplus t \cdot A^{0}\left(\mathbb{A}^{2} \backslash V, \mathrm{M}\right)
$$

and $\mathrm{m}^{*}\left\{27 x^{2}+4 y^{3}\right\}=12\{t\}+\left\{27 x^{2}+4 y^{3}\right\}$. Then an element $\left\{27 x^{2}+4 y^{3}\right\} \cdot \alpha$ is unramified if and only if $\alpha \in \mathrm{M}^{\bullet}(k)_{12}$.

Corollary 3.2. Assume that the characteristic of $k$ is different from 2,3 . We have

$$
{ }^{c} \operatorname{Br}\left(\mathcal{M}_{1,1}\right) \simeq{ }^{c} \operatorname{Br}(k) \oplus \mathrm{H}^{1}(k, \mathbb{Z} / 12 \mathbb{Z}) .
$$

Moreover, the group is generated by constant elements (i.e., pullbacks from ${ }^{c} \mathrm{Br}(k)$ ) and cyclic algebras.

Proof. The formula for the cohomological Brauer group follows immediately by applying Theorem 3.1 to $\mathrm{M}=\mathrm{H}_{\mu_{\ell}^{v}}$ for increasing $\ell$. Then Lemmas 2.18 and 2.19 allow us to conclude immediately.

\section{Some equivariant computations}

We begin by briefly recalling the Edidin-Graham-Totaro equivariant approximation construction. After that, we will explicitly compute some equivariant Chow groups with coefficients: these computations will be frequently used in the remainder of the paper.

Consider an algebraic space $X$ being acted upon by an affine, smooth algebraic group $G / k$. By Lemma 2.16 we know that for any $i$ there is a representation $V_{i}$ such that $G$ acts freely on an open subset $U_{i}$ whose complement has codimension at least $i$. Because the action of $G$ on $X \times U_{i}$ is free, the quotient $\left[X \times U_{i} / G\right]$ is an algebraic space.

Now, the map $\left[X \times U_{i} / G\right] \rightarrow[X / G]$ is the composition of a vector bundle and an open immersion whose complement has codimension at least $i$. Consider a collection of functors $F^{j}$ from the category of algebraic stacks over $k$ (or a subcategory, e.g., smooth stacks) to the category of groups such that the following are true:

○ If $E \rightarrow Y$ is a vector bundle, then $F^{j}(Y)=F^{j}(E)$.

- If $U \rightarrow Y$ is an open immersion whose complement has codimension $\geq i$, then $F^{j}(Y)=F^{j}(U)$ for every $j<i$.

Then we have $F^{j}([X / G])=F^{j}\left(\left[X \times U_{i} / G\right]\right)$ for some sufficiently large $i$. If we see $F^{\bullet}(\mathcal{X})=\oplus_{j} F^{j}(\mathcal{X})$ as a graded group, then $F^{\bullet}(\mathcal{X})=\oplus_{j} F^{j}\left(\left[X \times U_{i} / G\right]\right)$. An example of such a graded functor is étale cohomology, or singular cohomology when everything is defined over $\mathbb{C}$.

We can also go the other way around: if the theory $F$ is defined only for algebraic spaces, and it has the properties just described, then there is only one possible extension to quotient stacks that still satisfies the same properties - that is, we must have $F^{j}([X / G])=F^{j}\left(\left[X \times U_{i} / G\right]\right)$ for some large enough $i$.

A simple double fibration argument shows that this does not depend on the choice of the representation of $G$, and moreover this can be proven to be independent of the presentation as well, so it gives a welldefined extension of the graded functor $F^{\bullet}$ to quotient stacks $[X / G]$.

This construction, which already existed in the context of equivariant homology, was first used by Totaro [28] to compute the Chow rings of some classifying stacks B $G$, and was later extended by Edidin and Graham to general quotient stacks [10]. 
The construction carries over immediately to Chow groups with coefficients. Given an algebraic group $G$ acting on a scheme $X$, we will denote the $G$-equivariant Chow groups with coefficients of $X$ by

$$
\bigoplus_{i \geq 0} A_{G}^{i}(X, \mathrm{M})=A_{G}^{*}(X, \mathrm{M}) \text {. }
$$

This was first done by Guillot [17]. Note that contrary to the case of schemes, there is no maximum bound for the codimension of a nonzero element.

These groups enjoy all the same properties as the ordinary Chow groups with coefficients. Sometimes we will refer to the equivariant cycle groups $C_{G}^{i}(X, \mathrm{M})$; by this we will always mean the cycles on an appropriate equivariant approximation.

Cohomological invariants turn out to be equal to the 0-codimensional Chow group with coefficents for algebraic spaces, hence we can compute them on quotient stacks by using equivariant Chow groups with coefficients.

Proposition 4.1. Let $X$ be a smooth quasi-separated algebraic space over $k$, being acted upon by a smooth affine group $G / k$. Then

$$
\operatorname{Inv}^{\bullet}([X / G], \mathrm{M})=A_{G}^{0}(X, \mathrm{M}) .
$$

Proof. This is the content of [22, Thm. 4.16] in the case where the coefficients are in étale cohomology. The general case can be obtained in the exact same way, as an immediate consequence of Theorem 2.10 and Corollary 2.12.

In the rest of this section we will perform some explicit computations of equivariant Chow groups with coefficients. These results will be frequently used in the remainder of the paper.

Proposition 4.2. We have

$$
A_{\mathrm{GL}_{\mathrm{m}}}^{*}(\operatorname{Spec}(k), \mathrm{M})=\mathrm{CH}_{\mathrm{GL}_{\mathrm{m}}}^{*}(\operatorname{Spec}(k)) \otimes \mathrm{M}^{\bullet}(k) .
$$

Proof. Let $V$ be a finite-dimensional vector space and let $\mathrm{Gr}_{m}(V)$ be the Grassmannian of $m$-subspaces of $V$. We are going to prove that

$$
\mathrm{CH}^{i}\left(\mathrm{Gr}_{m}(V)\right) \otimes \mathrm{M}^{\bullet}(k) \simeq A^{i}\left(\mathrm{Gr}_{m}(V), \mathrm{M}\right)
$$

for every $i \geq 0$ and $0<m \leq \operatorname{dim}(V)$. This will readily imply the proposition, because of the isomorphisms

$$
\mathrm{CH}^{i}\left(\mathrm{Gr}_{m}(V)\right) \simeq \mathrm{CH}_{\mathrm{GL}_{\mathrm{m}}}^{i}(\operatorname{Spec}(k)), \quad A^{i}\left(\mathrm{Gr}_{m}(V), \mathrm{M}\right) \simeq A_{\mathrm{GL}_{\mathrm{m}}}^{i}(\operatorname{Spec}(k), \mathrm{M}),
$$

for vector spaces $V$ of sufficiently high dimension (the proof in [10, Subsec. 3.2] for classical Chow rings holds verbatim).

Let $\mathrm{Fl}_{m}(V)$ be the scheme of complete flags of length $m$ in $V$. Observe that $\mathrm{Fl}_{m}(V)$ can be constructed as a tower of projective bundles both over $\operatorname{Spec}(k)$ and $\operatorname{Gr}_{m}(V)$.

If the Chow ring of a smooth scheme $X$ is generated by Chern classes of vector bundles, thanks to Proposition 2.21 there is a well-defined multiplication morphism

$$
\mathrm{CH}^{i}(X) \otimes \mathrm{M}^{\bullet}(k) \rightarrow A^{i}(X, \mathrm{M}) .
$$

Moreover, if $f: Y \rightarrow X$ is a flat morphism of relative constant dimension from a smooth scheme whose Chow ring is also generated by Chern classes, it is easy to check that the pullback of a product is equal to the product of pullbacks. 
Applying this to the morphism $\mathrm{Fl}_{m}(V) \rightarrow \mathrm{Gr}_{m}(V)$, we obtain the following commutative diagram:

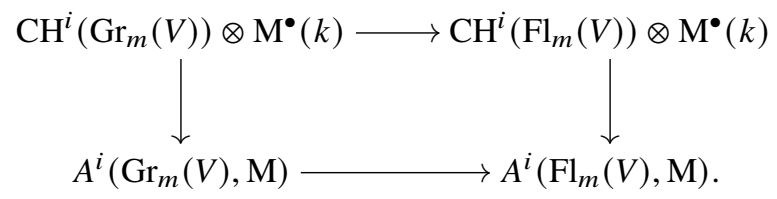

The fact that $\mathrm{Fl}_{m}(V)$ is a tower of projective bundles over a point, combined with an iterated application of [21, Prop. 2.4], shows that the right vertical morphism in the diagram is an isomorphism.

Define a splitting $s$ of the bottom horizontal arrow of diagram (4.1) as follows: at each level of the tower of projective bundles $\mathrm{Fl}_{m}(V) \rightarrow \mathrm{Gr}_{m}(V)$, multiply $r$ times with the appropriate hyperplane section (here $r$ is the dimension of the fibre at that level) and then take the push-forward to the level below. In the same way, we can construct a splitting $s^{\prime}$ at the level of Chow groups.

The pullback $f^{*}: \mathrm{CH}^{*}\left(\mathrm{Gr}_{m}(V)\right) \rightarrow \mathrm{CH}^{*}\left(\mathrm{Fl}_{m}(V)\right)$ is then split injective, and from this it is easy to deduce that the top horizontal arrow in diagram (4.1) is injective, and hence the left vertical arrow must be injective as well.

To show surjectivity, observe that if $\alpha$ is an element in $A^{*}\left(\mathrm{Gr}_{m}(V), \mathrm{M}\right)$, we have

$$
\alpha=s\left(f^{*}(\alpha)\right)=s\left(\sum \xi_{i} \cdot \beta_{i}\right)=\sum s^{\prime}\left(\xi_{i}\right) \cdot \beta_{i}
$$

for some $\xi_{i}$ in $\mathrm{CH}^{*}\left(\mathrm{Fl}_{m}(V)\right)$ and $\beta_{i}$ in $\mathrm{M}^{\bullet}(k)$. Therefore the left vertical morphism of equation (4.1) is surjective and this concludes the proof.

Proposition 4.3. We have

$$
A_{\mu_{\ell}}^{*}(\operatorname{Spec}(k), \mathrm{M})=(\mathbb{Z}[s] / \ell s) \otimes \mathrm{M}^{\bullet}(k) \underset{n}{\oplus} s^{n} \alpha \cdot \mathbf{M}^{\bullet}(k)_{\ell},
$$

where s has codimension 1 and cohomological degree 0 and $\alpha$ has codimension 0 and cohomological degree 1 . In particular, if $M$ is $\ell$-torsion,

$$
A_{\mu_{\ell}}^{*}(\operatorname{Spec}(k), \mathrm{M}) \simeq A_{\mu_{\ell}}^{*}\left(\operatorname{Spec}(k), \mathrm{H}_{\mathbb{Z} / \ell \mathbb{Z})}\right) \mathrm{M}^{\bullet}(k) .
$$

Proof. We have $\mathrm{B} \mu_{\ell}=\left[\left(\mathbb{A}^{1} \backslash\{0\}\right) / \mathbb{G}_{m}\right]$, where the action is defined as $\lambda \cdot x=\lambda^{\ell} x$.

The localisation long exact sequence for the $\mathbb{G}_{m}$-equivariant embedding $\{0\} \hookrightarrow \mathbb{A}^{1}$ reads as follows:

$$
\cdots \rightarrow A_{\mathbb{G}_{m}}^{i}\left(\mathbb{A}^{1}, \mathrm{M}\right) \rightarrow A_{\mathbb{G}_{m}}^{i}\left(\mathbb{A}^{1} \backslash\{0\}, \mathrm{M}\right) \rightarrow A_{\mathbb{G}_{m}}^{i}(\{0\}, \mathrm{M}) \rightarrow A_{\mathbb{G}_{m}}^{i+1}\left(\mathbb{A}^{1}, \mathrm{M}\right) \rightarrow \cdots
$$

By Proposition 4.2, we have

$$
A_{\mathbb{G}_{m}}^{i}\left(\mathbb{A}^{1}, \mathrm{M}\right) \simeq A_{\mathbb{G}_{m}}^{i}(\{0\}, \mathrm{M}) \simeq \mathrm{M}^{\bullet}(k) s^{i}
$$

Moreover by Proposition 2.21 the push-forward $i_{*}: A_{\mathbb{G}_{m}}^{i}(\{0\}, \mathrm{M}) \rightarrow A_{\mathbb{G}_{m}}^{i+1}\left(\mathbb{A}^{1}, \mathrm{M}\right)$ corresponds to multiplication by $[\{0\}]=\ell s$.

Proposition 4.2 assures us that multiplying by $s$ defines an injective morphism, hence we deduce

$$
\operatorname{ker}\left(i_{*}\right)=\mathrm{M}^{\bullet}(k)_{\ell} \cdot s^{i}, \quad \operatorname{im}\left(i_{*}\right)=\mathrm{M}^{\bullet}(k) \cdot \ell s^{i+1} .
$$

It follows that for every $i \geq 0$ we have the following short exact sequence:

$$
0 \rightarrow(\mathbb{Z}[s] / \ell s) \otimes \mathrm{M}^{\bullet}(k) \rightarrow A_{\mathbb{G}_{m}}^{i}\left(\mathbb{A}^{1} \backslash\{0\}, \mathrm{M}\right) \rightarrow \mathrm{M}^{\bullet}(k)_{\ell} \cdot s^{i} \rightarrow 0 .
$$


Recall from Lemma 2.22 that there is a splitting

$$
A^{i}(\{0\}, \mathrm{M}) \rightarrow A^{i}\left(\mathbb{A}^{1} \backslash\{0\}, \mathrm{M}\right)
$$

given by multiplication by the element $\{t\} \in A^{0}\left(\mathbb{A}^{1} \backslash\{0\}, \mathrm{K}_{\mathrm{M}}^{1}\right)$.

If we consider $\{t\} \cdot \tau$, where $\tau \in \mathbf{M}^{\bullet}(k)$ is an $\ell$-torsion element, this element is invariant for the $\mathbb{G}_{m^{-}}$ action, as the action sends $\{t\}$ to $\left\{\lambda^{\ell} t\right\}=\ell\{\lambda\}+\{t\}$. Thus it glues to an element $\gamma \in A_{\mathbb{G}_{m}}^{0}\left(\mathbb{A}^{1} \backslash\{0\}, \mathrm{M}\right)$, splitting the exact sequence for $i=0$.

Combining this splitting with multiplication by $s^{i}$, we obtain a splitting for every codimension $i$, proving our claim.

Proposition 4.4. Let $\mathrm{M}$ be a torsion cycle module, and let $\mu_{\ell}$ act trivially on a scheme $X$. Then

$$
A_{\mu_{\ell}}^{*}(X, \mathrm{M}) \simeq(\mathbb{Z}[s] / \ell s) \otimes A^{*}(X, \mathrm{M}) \oplus_{n \geq 0} s^{n} \alpha A^{*}(X, \mathrm{M})_{\ell}
$$

In particular,

$$
A_{\mu_{\ell}}^{0}(X, \mathrm{M})=A^{0}(X, \mathrm{M}) \oplus \alpha A^{0}(X, \mathrm{M})_{\ell}
$$

Proof. Note that if $\mathbb{G}_{m}$ acts trivially on $X$, then $A_{\mathbb{G}_{m}}^{i}(X, \mathrm{M})=A^{i}\left(X \times \mathbb{P}_{k}^{r}, \mathrm{M}\right)$ for some $r>i$, showing that

$$
A_{\mathbb{G}_{m}}^{*}(X, \mathrm{M})=A^{*}(X, \mathrm{M})[s]=\mathrm{CH}_{\mathbb{G}_{m}}^{*}(\operatorname{Spec}(k)) \otimes A^{*}(X, \mathrm{M}) .
$$

Using this, we can just repeat the proof of the previous Proposition 4.3 using the $\ell$-twisted action of $\mathbb{G}_{m}$ on $X \times \mathbb{G}_{m}$ and the compatibility of the pullback

$$
A_{\mathbb{G}_{m}}^{*}(X, \mathrm{M}) \rightarrow A_{\mathbb{G}_{m}}^{*}\left(X \times \mathbb{G}_{m}, \mathrm{M}\right)
$$

with the long exact sequence.

Proposition 4.5. Let $\mathrm{M}$ be an odd torsion cycle module. Then

$$
A_{\mathrm{PGL}_{2}}^{*}(\operatorname{Spec}(k), \mathrm{M})=\mathrm{CH}_{\mathrm{PGL}_{2}}^{*}(\operatorname{Spec}(k)) \otimes \mathrm{M}^{\bullet}(k) .
$$

The strategy of proof is the following: we first compute $A^{*}\left(\mathrm{O}_{\mathrm{n}}(\operatorname{Spec}(k)), \mathrm{M}\right)$ for $n=1,2,3$, and after that we exploit the isomorphism $\mathrm{PGL}_{2} \simeq \mathrm{SO}_{3}$ to conclude our computation.

The proof is borrowed almost verbatim from [23, Cor. 1.10], although we have to deal here with a generic torsion cycle module $\mathrm{M}$, which in particular may not possess a pairing.

Lemma 4.6. Let $V$ be an $n$-dimensional vector space endowed with a nondegenerate quadratic form $q$. Set $\mathrm{O}_{\mathrm{n}}=O(V, q)$. Let $\mathrm{M}$ be an odd torsion cycle module. Then we have the following:

○ $A_{\mathrm{O}_{1}}^{*}(\operatorname{Spec}(k), \mathrm{M})=\mathrm{M}^{\bullet}(k)$.

$\circ A_{\mathrm{O}_{2}}^{*}(\operatorname{Spec}(k), \mathrm{M}) \simeq \mathrm{M}^{\bullet}(k)\left[c_{2}\right] \simeq \mathrm{CH}_{\mathrm{O}_{2}}^{*}(\operatorname{Spec}(k)) \otimes \mathrm{M}^{\bullet}(k)$.

$\circ A_{\mathrm{O}_{3}}^{*}(\operatorname{Spec}(k), \mathrm{M}) \simeq \mathrm{M}^{\bullet}(k)\left[c_{2}\right] \simeq \mathrm{CH}_{\mathrm{O}_{3}}^{*}(\operatorname{Spec}(k)) \otimes \mathrm{M}^{\bullet}(k)$.

Proof. Let $\mathrm{M}$ be an odd torsion cycle module. Using the description of $A_{\mu_{2}}^{*}(\operatorname{Spec}(k), \mathrm{M})$ given by Proposition 4.3, we see that every element of codimension $>0$ is of both 2 -torsion and $\ell$-torsion, hence 0 . We deduce

$$
A_{\mathrm{O}_{1}}^{*}(\operatorname{Spec}(k), \mathrm{M}) \simeq A_{\mu_{2}}^{*}(\operatorname{Spec}(k), \mathrm{M}) \simeq \mathrm{M}^{\bullet}(k) .
$$

Define $C \subset V \backslash\{0\}$ as the vanishing locus of $q$. Let $B$ be the complement of $C$ in $V \backslash\{0\}$. All these schemes are $\mathrm{O}_{\mathrm{n}}$-invariant. 
We can compute $A_{\mathrm{O}_{\mathrm{n}}}^{*}(B, \mathrm{M})$ as follows: consider the morphism $B \rightarrow \mathbb{G}_{m}$ induced by $q$ and form the Cartesian square

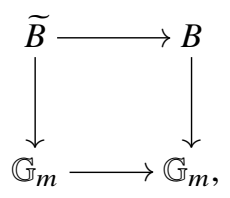

where the bottom morphism is the square map. We have $\widetilde{B} \simeq Q \times \mathbb{G}_{m}$, where $Q \subset B$ is the fibre over the unit in $\mathbb{G}_{m}$, and moreover both horizontal arrows are $\mu_{2}$-torsors. Therefore

$$
A_{\mathrm{O}_{n} \times \mu_{2}}^{*}\left(Q \times \mathbb{G}_{m}, \mathrm{M}\right) \simeq A_{\mathrm{O}_{n}}^{*}(B, \mathrm{M})
$$

Let $\mathcal{L}$ be the $\left(\mathrm{O}_{\mathrm{n}} \times \mu_{2}\right)$-equivariant line bundle over $Q$ such that the equivariant $\mathbb{G}_{m}$-torsor $Q \times \mathbb{G}_{m} \rightarrow Q$ is the complement in $\mathcal{L}$ of the zero section. The associated localisation exact sequence is

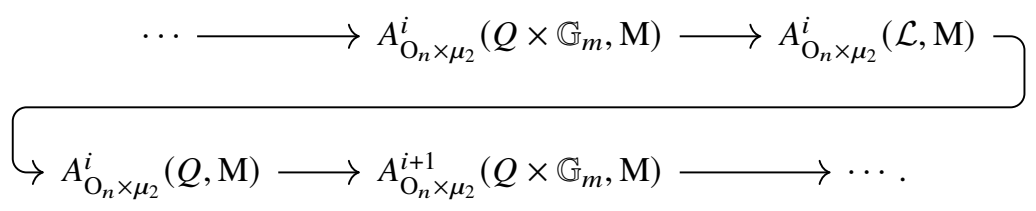

Via the identification $A_{\mathrm{O}_{\mathrm{n}} \times \mu_{2}}^{*}(\mathcal{L}, \mathrm{M}) \simeq A_{\mathrm{O}_{\mathrm{n}} \times \mu_{2}}^{*}(Q, \mathrm{M})$, we see that the last map is given by multiplication by $c_{1}^{\mathrm{O}_{\mathrm{n}} \times \mu_{2}}(\mathcal{L})$.

The action of $\mathrm{O}_{\mathrm{n}} \times \mu_{2}$ on $Q$ is transitive: its stabiliser is $\mathrm{O}_{\mathrm{n}-1} \times \mu_{2}$, hence $\left[Q / \mathrm{O}_{\mathrm{n}}\right] \simeq B\left(\mathrm{O}_{\mathrm{n}-1} \times \mu_{2}\right)$ and

$$
A_{\mathrm{O}_{\mathrm{n}} \times \mu_{2}}^{*}(Q, \mathrm{M}) \simeq A_{\mathrm{O}_{n-1} \times \mu_{2}}^{*}(\operatorname{Spec}(k), \mathrm{M}) \simeq A_{\mathrm{O}_{\mathrm{n}-1}}^{*}(\operatorname{Spec}(k), \mathrm{M}),
$$

where the last isomorphism is a consequence of the triviality of $A_{\mu_{2}}^{*}(\operatorname{Spec}(k), \mathrm{M})$.

By Proposition 2.21, we have

$$
c_{1}^{\mathrm{O}_{\mathrm{n}} \times \mu_{2}}(\mathcal{L})(\alpha)=c_{1}^{\mathrm{O}_{\mathrm{n}} \times \mu_{2}}(\mathcal{L})(1) \cdot \alpha
$$

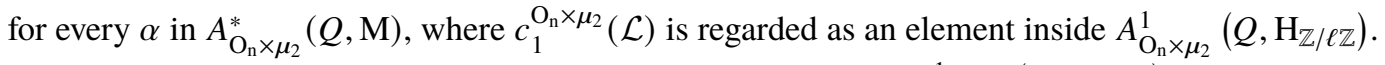

Observe that from formula (4.5), it follows that the part of $A_{\mathrm{O}_{\mathrm{n}} \times \mu_{2}}^{1}\left(Q, \mathrm{H}_{\mathbb{Z} / \ell \mathbb{Z}}\right)$ of cohomological degree 0 is equal to $\mathbb{Z} / \ell \mathbb{Z} \cdot c_{1} /\left\langle 2 \cdot c_{1}\right\rangle=0$. Therefore, from sequence (4.4) we deduce the exact sequence

$$
0 \rightarrow A_{\mathrm{O}_{\mathrm{n}} \times \mu_{2}}^{i}(Q, \mathrm{M}) \rightarrow A_{\mathrm{O}_{\mathrm{n}} \times \mu_{2}}^{i}\left(Q \times \mathbb{G}_{m}, \mathrm{M}\right) \rightarrow A_{\mathrm{O}_{n} \times \mu_{2}}^{i}(Q, \mathrm{M}) \rightarrow 0 .
$$

Combining this with the content of formulas (4.5) and (4.3), we get

$$
A_{\mathrm{O}_{n}}^{*}(B, \mathrm{M}) \simeq A_{\mathrm{O}_{n-1}}^{*}(\operatorname{Spec}(k), \mathrm{M}) \oplus A_{\mathrm{O}_{n-1}}^{*}(\operatorname{Spec}(k), \mathrm{M}) \cdot \beta
$$

as $\mathrm{H}_{\mathbb{Z} / \ell \mathbb{Z}}^{\bullet}(k)$-modules, where $\beta$ has codimension 0 and cohomological degree 1 .

Next we compute $A_{\mathrm{O}_{\mathrm{n}}}^{*}(C, \mathrm{M})$, where $C$ is the vanishing locus of $q$ in $V \backslash\{0\}$. The action of $\mathrm{O}_{\mathrm{n}}$ on $C$ is transitive: the stabiliser is given by the semidirect product of $\mathrm{O}_{\mathrm{n}-2}$ with a subgroup $H$ of the group of affine transformations of a vector space $W$. More precisely, the group $H$ is isomorphic to $W$ via the natural projection of $\operatorname{Aff}(V)$ on translations. It follows from [23, Lemma 1.7] that

$$
A_{\mathrm{O}_{n}}^{*}(C, \mathrm{M}) \simeq A_{\mathrm{O}_{\mathrm{n}-2} \ltimes H}^{*}(\operatorname{Spec}(k), \mathrm{M}) \simeq A_{\mathrm{O}_{\mathrm{n}-2}}^{*}(\operatorname{Spec}(k), \mathrm{M}) .
$$


Next we compute $A_{\mathrm{O}_{2}}^{*}(\operatorname{Spec}(k), \mathrm{M})$. First observe that $O_{0}=\{i d\}$ and $\mathrm{O}_{1} \simeq \mu_{2}$. Consider the pushforward morphism

$$
i_{*}: A_{\mathrm{O}_{2}}^{*}(C, \mathrm{M}) \simeq \mathrm{M}^{\bullet}(k) \rightarrow A_{\mathrm{O}_{2}}^{*+1}(V \backslash\{0\}, \mathrm{M}) .
$$

The isomorphism on the left side comes from formula (4.7). By the projection formula, $i_{*} \alpha=\alpha \cdot i_{*}(1)$, and $i_{*}(1)$ is in degree 0 and codimension 1 . We have $A_{\mathrm{O}_{2}}^{1}(V \backslash\{0\}, \mathrm{M}) \simeq A_{\mathrm{O}_{2}}^{1}(V, \mathrm{M})$, which is in turn isomorphic to $A_{\mathrm{O}_{2}}^{1}(\operatorname{Spec}(k), \mathrm{M})$.

The piece of the latter group of cohomological degree 0 turns out to be equal to $\mathrm{CH}_{\mathrm{O}_{2}}^{1}(\mathrm{Spec}(k)) \otimes$ $M^{0}(k)$, which vanishes because it is of both 2-torsion and $\ell$-torsion. We deduce that $i_{*}=0$.

This last remark, applied to the localisation exact sequence for $C \subset V \backslash\{0\}$, implies that the following short sequence is exact:

$$
0 \rightarrow A_{\mathrm{O}_{2}}^{*}(V \backslash\{0\}, \mathrm{M}) \rightarrow A_{\mathrm{O}_{2}}^{*}(B, \mathrm{M}) \rightarrow A_{\mathrm{O}_{2}}^{*}(C, \mathrm{M}) \rightarrow 0 .
$$

By formula (4.7), the group on the right is equal to $\mathbf{M}^{\bullet}(k)$ in codimension 0 and is 0 in higher codimension. By formula (4.6), the group in the middle is equal to $A_{\mu_{2}}^{*}(\operatorname{Spec}(k), \mathrm{M}) \oplus A_{\mu_{2}}^{*}(\operatorname{Spec}(k), \mathrm{M})$. $\beta$ in codimension 0 and vanishes in higher codimension, where $\beta$ has cohomological degree 1 .

From formula (4.2) we know that $A_{\mu_{2}}^{*}(\operatorname{Spec}(k), \mathrm{M})$ is trivial, hence the short exact sequence (4.8) implies

$$
A_{\mathrm{O}_{2}}^{*}(V \backslash\{0\}, \mathrm{M}) \simeq \mathrm{M}^{\bullet}(k) .
$$

Now consider the localisation exact sequence

$$
\cdots \rightarrow A_{\mathrm{O}_{2}}^{i}(V, \mathrm{M}) \rightarrow A_{\mathrm{O}_{2}}^{i}(V \backslash\{0\}, \mathrm{M}) \rightarrow A_{\mathrm{O}_{2}}^{i-1}(\{0\}, \mathrm{M}) \rightarrow A_{\mathrm{O}_{2}}^{i+1}(V, \mathrm{M}) \rightarrow \cdots
$$

induced by the open embedding $V \backslash\{0\} \hookrightarrow V$.

Observe that $A_{\mathrm{O}_{2}}^{0}(V, \mathrm{M}) \simeq A_{\mathrm{O}_{2}}^{0}(V \backslash\{0\})$ because the codimension of $\{0\}$ in $V$ is $>1$. Moreover, from formula (4.9) we know that $A_{\mathrm{O}_{2}}^{i}(V \backslash\{0\}, \mathrm{M})=0$ for $i>0$. Observe also that the push-forward induced by the closed embedding $\{0\} \hookrightarrow V$ coincides with multiplication by $c_{2}^{\mathrm{O}_{2}}(V)=: c_{2}$.

Hence from the long exact sequence (4.10) we readily deduce the following:

○ $A_{\mathrm{O}_{2}}^{0}(V, \mathrm{M}) \simeq \mathrm{M}^{\bullet}(k)$.

○ $A_{\mathrm{O}_{2}}^{i}(V, \mathrm{M})=0$ for $i$ odd.

○ $A_{\mathrm{O}_{2}}^{i+2}(V, \mathrm{M}) \simeq c_{2} \cdot A_{\mathrm{O}_{2}}^{i}(V, \mathrm{M})$.

Therefore

$$
A_{\mathrm{O}_{2}}^{*}(\operatorname{Spec}(k), \mathrm{M}) \simeq \mathrm{M}^{\bullet}(k)\left[c_{2}\right] \simeq \mathrm{CH}_{\mathrm{O}_{2}}^{*}(\operatorname{Spec}(k)) \otimes \mathrm{M}^{\bullet}(k) .
$$

Next we prove that the same result holds for $O_{3}$. As before, we start by computing $A_{\mathrm{O}_{3}}^{*}(V \backslash\{0\}, \mathrm{M})$.

The triviality of $A_{\mu_{2}}^{*}(\operatorname{Spec}(k), \mathrm{M})$ together with formula (4.7) implies that $A_{\mathrm{O}_{3}}^{*}(C, \mathrm{M}) \simeq \mathrm{M}^{\bullet}(k)$. The same argument used for $\mathrm{O}_{2}$ shows that the push-forward morphism $i_{*}: A_{\mathrm{O}_{3}}^{*}(C, \mathrm{M}) \rightarrow A_{\mathrm{O}_{3}}^{*}(V \backslash\{0\}, \mathrm{M})$ is zero.

Plugging this information into the localisation exact sequence induced by the open embedding $B \hookrightarrow V \backslash\{0\}$, we deduce that $A_{\mathrm{O}_{3}}^{i}(V \backslash\{0\}, \mathrm{M}) \simeq A_{\mathrm{O}_{3}}^{i}(B, \mathrm{M})$ for $i>0$ and that the following is exact:

$$
0 \rightarrow A_{\mathrm{O}_{3}}^{0}(V \backslash\{0\}, \mathrm{M}) \rightarrow A_{\mathrm{O}_{3}}^{0}(B, \mathrm{M}) \rightarrow A_{\mathrm{O}_{3}}^{0}(C, \mathrm{M}) \simeq \mathrm{M}^{\bullet}(k) \rightarrow 0 .
$$

By formula (4.6) together with formula (4.11), we have

$$
A_{\mathrm{O}_{3}}^{*}(B, \mathrm{M}) \simeq \mathrm{M}^{\bullet}(k)\left[c_{2}\right] \oplus \mathrm{M}^{\bullet}(k)\left[c_{2}\right] \cdot \beta,
$$


with $\beta$ of codimension 0 and cohomological degree 1 . We deduce that

$$
A_{\mathrm{O}_{3}}^{*}(V \backslash\{0\}, \mathrm{M}) \simeq \mathrm{M}^{\bullet}(k)\left[c_{2}\right] \oplus \mathbf{M}^{\bullet}(k)\left[c_{2}\right] \cdot \gamma,
$$

where $\gamma$ has codimension 2 and cohomological degree 1 .

Observe that the push-forward morphism $A_{\mathrm{O}_{3}}^{*}(\{0\}, \mathrm{M}) \rightarrow A_{\mathrm{O}_{3}}^{*+3}(V, \mathrm{M})$ coincides with multiplication by $c_{3}(1)$, which is both a 2 -torsion and an $\ell$-torsion element, hence zero.

Consider now the open embedding $V \backslash\{0\} \hookrightarrow V$. By what we have just proved, the following short sequences are exact for every $i$ :

$$
0 \rightarrow A_{\mathrm{O}_{3}}^{i}(V, \mathrm{M}) \rightarrow A_{\mathrm{O}_{3}}^{i}(V \backslash\{0\}, \mathrm{M}) \rightarrow A_{\mathrm{O}_{3}}^{i-2}(\{0\}, \mathrm{M}) \rightarrow 0
$$

We immediately deduce the following:

○ $A_{\mathrm{O}_{3}}^{0}(V, \mathrm{M}) \simeq \mathrm{M}^{\bullet}(k)$.

- $A_{\mathrm{O}_{3}}^{i}(V, \mathrm{M})=0$ for $i$ odd.

○ $A_{\mathrm{O}_{3}}^{2 i}(V, \mathrm{M}) \simeq \mathrm{M}^{\bullet}(k) \cdot c_{2}^{i}$.

Therefore

$$
A_{\mathrm{O}_{3}}^{*}(\operatorname{Spec}(k), \mathrm{M}) \simeq \mathrm{M}^{\bullet}(k)\left[c_{2}\right] \simeq \mathrm{CH}_{\mathrm{O}_{3}}^{*}(\operatorname{Spec}(k)) \otimes \mathrm{M}^{\bullet}(k),
$$

which concludes the proof of the lemma.

Proof of Proposition 4.5. Observe that $\mathrm{PGL}_{2} \simeq \mathrm{SO}_{3}$ and $O_{3} \simeq \mathrm{SO}_{3} \times \mu_{2}$. The result is then a direct consequence of Proposition 4.4 and Lemma 4.6.

\section{Generalised cohomological invariants of some classical groups}

We begin this section by discussing the relations between the cycle modules $\mathrm{H}_{\mathbb{Z} / \ell \mathbb{Z}}=\mathrm{K}_{\ell}$ as $\ell$ varies, and showing that the $\ell$-torsion $\operatorname{Inv}^{\bullet}(\mathcal{X}, \mathrm{M})_{\ell}$ has a natural $\operatorname{Inv}^{\bullet}\left(\mathcal{X}, \mathrm{K}_{\ell}\right)$ module structure. This will be crucial in the rest of the paper. Using these ideas, we will extend some results on classical cohomological invariants from [15] to work with coefficients in an arbitrary cycle module; in particular, we will compute the generalised invariants of $\mathrm{S}_{\mathrm{n}}$ and $\mathrm{PGL}_{2}$, which will be necessary later on.

For the remainder of this section, by $\eta$ we will always mean a positive integer not divisible by $\operatorname{char}(k)$. Consider the exact sequence

$$
0 \rightarrow \mathbb{Z} / \eta \mathbb{Z} \rightarrow \mathbb{Z} / \ell \eta \mathbb{Z} \rightarrow \mathbb{Z} / \ell \mathbb{Z} \rightarrow 0
$$

We can twist it to get exact sequences

$$
0 \rightarrow \mathbb{Z} / \eta \mathbb{Z}(i) \rightarrow \mathbb{Z} / \ell \eta \mathbb{Z}(i) \rightarrow \mathbb{Z} / \ell \mathbb{Z}(i) \rightarrow 0
$$

In particular, when $i=1$ we retrieve the exact sequence

$$
0 \rightarrow \mu_{\eta} \rightarrow \mu_{\ell \eta} \rightarrow \mu_{\ell} \rightarrow 0
$$

Consequently, for each $i$ we have an exact sequence

$$
\mathrm{H}^{i}(-, \mathbb{Z} / \eta \mathbb{Z}(i)) \rightarrow \mathrm{H}^{i}(-, \mathbb{Z} / \ell \eta \mathbb{Z}(i)) \rightarrow \mathrm{H}^{i}(-, \mathbb{Z} / \ell \mathbb{Z}(i))
$$

these maps form an exact sequence of cycle modules

$$
\mathrm{H}_{\mathbb{Z} / \eta \mathbb{Z}} \rightarrow \mathrm{H}_{\mathbb{Z} / \ell \eta \mathbb{Z}} \rightarrow \mathrm{H}_{\mathbb{Z} / \ell \mathbb{Z}}
$$


Using Voevodsky's norm-residue isomorphism [29, Thm. 6.1], we conclude that this is just the exact sequence

$$
\mathrm{K}_{\eta} \rightarrow \mathrm{K}_{\eta \ell} \rightarrow \mathrm{K}_{\ell} \rightarrow 0
$$

In particular, the last map is surjective, and the kernel is just the image of the kernel of $\mathrm{K}_{\mathrm{Mil}} \rightarrow \mathrm{K}_{\ell}-$ that is, the ideal $(\ell)$.

Lemma 5.1. Let $X / k$ be a smooth scheme, and set $\alpha \in \mathrm{K}_{\ell}(k(X))$. Let $\alpha^{\prime}$ be an inverse image of $\alpha$ in $\mathrm{K}_{\mathrm{Mil}}^{\bullet}(k(X))\left(\right.$ resp., $\left.\mathrm{K}_{\ell \eta}^{\bullet}(k(X))\right)$. If $\alpha$ is unramified, then $\alpha^{\prime} \cdot x$ is unramified for any $x \in$ $A^{0}\left(X, \mathrm{~K}_{\mathrm{Mil}}\right)_{\ell}\left(\right.$ resp., $\left.A^{0}\left(X, \mathrm{~K}_{\ell \eta}\right)_{\ell}\right)$. Moreover, the class $\alpha^{\prime} \cdot x$ does not depend on the choice of $\alpha^{\prime}$.

Proof. By the compatibility of the morphism of cycle modules with the boundary map, the ramification of $\alpha^{\prime}$ at any point $x$ of codimension 1 has to belong to the kernel of $\mathrm{K}_{\mathrm{Mil}}^{\bullet}(k(x)) \rightarrow \mathrm{K}_{\ell}^{\bullet}(k(x))$, which means it is a multiple of $\ell$, so $\alpha^{\prime} \cdot x$ is unramified as $\partial_{v}\left(\alpha^{\prime} \cdot x\right)=\partial_{v}\left(\alpha^{\prime}\right) \cdot x=0$. By the same reasoning, note that if $\alpha^{\prime \prime}$ is another inverse image of $\alpha$, the difference $\alpha^{\prime}-\alpha^{\prime \prime}$ has to be a multiple of $\ell$, concluding the proof.

Using this, we can define a multiplicative action of the invariants with coefficients in $\mathrm{K}_{\ell}$ on the $\ell$-torsion of the invariants with coefficients in any cycle module.

Lemma 5.2. Let $X / k$ be a smooth scheme endowed with a G-action. There is a well-defined multiplication

$$
A_{G}^{0}\left(X, \mathrm{~K}_{\ell}\right) \otimes A_{G}^{0}(X, \mathrm{M})_{\ell} \rightarrow A_{G}^{0}(X, \mathrm{M})_{\ell}
$$

which for any $G$-equivariant morphism $f: X \rightarrow Y$ of smooth $G$-schemes satisfies the formula $f^{*}(\alpha \cdot \beta)=$ $f^{*} \alpha \cdot f^{*} \beta$.

Proof. For the sake of simplicity, we give a proof in the nonequivariant setting. The general case can be deduced in the same way using equivariant approximation.

Let $\alpha$ be an element of $A^{0}\left(X, \mathrm{~K}_{\ell}\right) \subset \mathrm{K}_{\ell}^{\bullet}(k(X))$ and let $\beta$ be an element of $A_{G}^{0}(X, \mathrm{M})_{\ell}$. For every inverse image $\alpha^{\prime}$ of $\alpha$ in $\mathrm{K}_{\mathrm{Mil}}^{\bullet}(k(X))$, we have a well-defined product $\alpha^{\prime} \cdot \beta$ in $\mathrm{M}^{\bullet}(k(X))$ : this product is unramified on $X$ because the ramification of $\alpha^{\prime}$ at any point of codimension 1 is a multiple of $\ell$, just as in the proof of the Lemma 5.1.

By the same reasoning, the definition of the product does not depend on the choice of an inverse image $\alpha^{\prime}$. The compatibility with the pullback follows from the compatibility of the product $A^{0}\left(X, \mathrm{~K}_{\mathrm{Mil}}\right) \otimes$ $A^{0}(X, \mathrm{M}) \rightarrow A^{0}(X, \mathrm{M})$.

This allows us to easily describe the cohomological invariants of $\mu_{\ell}^{r}$ :

Lemma 5.3. The cohomological invariants of $\mathrm{B}\left(\mu_{\ell}^{r}\right)$ with coefficients in $\mathrm{M}$ are given by

$$
\left.\operatorname{Inv}^{\bullet}\left(\mathrm{B}\left(\mu_{\ell}^{r}\right), \mathrm{M}\right) \simeq \mathrm{M}^{\bullet}(k) \oplus \underset{J \subseteq\{1, \ldots, r\}}{\oplus} \alpha_{J} \cdot \mathrm{M}^{\bullet}(k)_{\ell}\right),
$$

where $\alpha_{\{j\}}$ is the pullback of the identity invariant through the projection

$$
\operatorname{Pr}_{j}: \mu_{\ell}^{r} \rightarrow \mu_{\ell}
$$

and if $J=\left\{j_{1}, \ldots, j_{m}\right\}$, then $\alpha_{J}=\alpha_{\left\{j_{1}\right\}} \cdots \alpha_{\left\{j_{m}\right\}}$.

In particular, if $\mathrm{M}$ has no $\ell$-torsion, the normalised invariants of $\mu_{\ell}^{r}$ with coefficients in $\mathrm{M}$ are trivial.

Proof. Consider the 2-dimensional $\mu_{\ell}$-representation $V$ given by diagonal matrices. The representation is free outside of 0 , so $V / \mu_{\ell}$ is an equivariant approximation of $\mathrm{B}\left(\mu_{\ell}\right)$, and $V^{i} / \mu_{\ell}^{i}$ is an equivariant approximation of $\mathrm{B}\left(\mu_{\ell}^{i}\right)$. 
Our claim is true for $r=1$ by Proposition 4.3. Assume by induction that it is true for $r-1$; then it follows immediately by applying Proposition 4.4 to $V^{r-1} / \mu_{\ell}^{r-1}$.

The following simple principle will be the key to extending many known computations of cohomological invariants with coefficients in $\mathrm{K}_{\ell}$ to arbitrary cycle modules:

Lemma 5.4. Let $\mathcal{X}$ be a smooth algebraic stack, and let $\phi: \mathrm{B} \mu_{\ell}^{r} \rightarrow \mathcal{X}$ be a morphism (e.g., the morphism $\mathrm{B} \mu_{\ell}^{r} \rightarrow \mathrm{B} G$ induced by a morphism $\mu_{\ell}^{r} \rightarrow G$ of group schemes). Let $G$ be a finite group acting on $\{1, \ldots, r\}$. Assume the pullback $\phi^{*}$ gives us an injection

$$
\operatorname{Inv}^{\bullet}(\mathcal{X}, \mathrm{M}) \subseteq \operatorname{Inv}^{\bullet}\left(\mu_{\ell}^{r}, \mathrm{M}\right)^{G}
$$

for every M, and consequently an injection on normalised invariants. Then the following are equivalent:

○ $\operatorname{Inv}^{\bullet}(\mathcal{X}, \mathrm{M})=\operatorname{Inv} \bullet\left(\mu_{\ell}^{r}, \mathrm{M}\right)^{G}$ for all $\mathrm{M}$.

$\circ \operatorname{Inv}^{\bullet}\left(\mathcal{X}, \mathrm{K}_{\ell}\right)=\operatorname{Inv}^{\bullet}\left(\mu_{\ell}^{r}, \mathrm{~K}_{\ell}\right)^{G}$.

Proof. Obviously the first point implies the second. We claim that the converse is also true. We have a commutative square

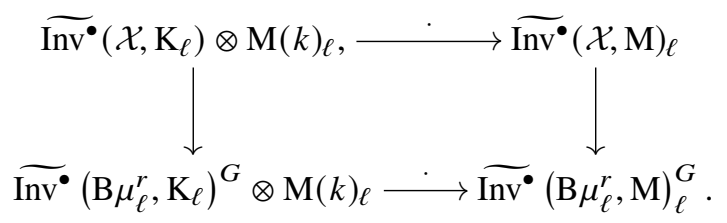

The left-hand vertical map is surjective by hypothesis. The bottom horizontal map is surjective as well; it can be easily seen from the structure of $\operatorname{Inv}{ }^{\bullet}\left(\mathrm{B} \mu_{l}^{r}, \mathrm{M}\right)$ that the $G$-invariant elements are generated by those in the form

$$
\alpha_{J}+\alpha_{g_{1}(J)}+\cdots+\alpha_{g_{m}(J)},
$$

where $\left\{J, g_{1}(J), \ldots, g_{m}(J)\right\}$ is the orbit of the subset $J \subset\{1, \ldots, r\}$, and these elements belong to the image of the bottom horizontal map. Then the right-hand vertical map is injective (by hypothesis) and surjective. This implies that the map on nonnormalised cohomological invariants is surjective as well, proving our claim.

Now, let $U_{n} \subset \mathrm{GL}_{n}$ be the subscheme of symmetric matrices. Consider the commutative diagram

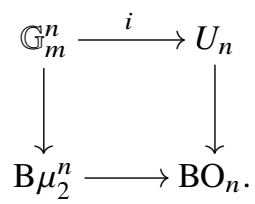

The vertical maps are given, respectively, by the quotient by $\mathbb{G}_{m}^{n}$ acting on itself with weight 2 and the quotient by $\mathrm{GL}_{n}$ acting by $(A, S) \rightarrow A^{\mathrm{T}} S A$. In particular, we can see the action of $\mathbb{G}_{m}^{n}$ on itself as the subgroup of diagonal matrices of $\mathrm{GL}_{n}$ acting on $\mathbb{G}_{m}^{n} \subset U_{n}$. The bottom map comes from the inclusion of the diagonal matrices with coefficients \pm 1 into $\mathrm{O}_{\mathrm{n}}$. Note that both vertical maps are quotients by special groups and thus smooth Nisnevich.

It is a well-known fact that in characteristic different from 2, every symmetric matrix is equivalent to a diagonal matrix under the action of $\mathrm{GL}_{n}$. The map $U_{n} \rightarrow \mathrm{BO}_{n}$ is smooth Nisnevich because $\mathrm{GL}_{n}$ is special. This shows that given any point $p \in \mathrm{BO}_{n}$, there is a lifting of $p$ to $\mathbb{G}_{m}^{n}$ - that is, the map $G_{m}^{n} \rightarrow \mathrm{BO}_{n}$ is smooth Nisnevich. Consequently, the map from $\mathrm{B} \mu_{2}^{n}$ to $\mathrm{BO}_{n}$ must be smooth Nisnevich as well. 
Proposition 5.5. Any normalised cohomological invariant of $\mathrm{O}_{\mathrm{n}}$ is of 2-torsion. We have

$$
\operatorname{Inv}^{\bullet}\left(\mathrm{BO}_{n}, \mathbf{M}\right)=\mathbf{M}^{\bullet}(k) \oplus \alpha_{1} \cdot \mathbf{M}^{\bullet}(k)_{2} \oplus \cdots \oplus \alpha_{n} \cdot \mathbf{M}^{\bullet}(k)_{2},
$$

where $\alpha_{i}$ is the ith symmetric function in $\alpha_{\{1\}}, \ldots, \alpha_{\{n\}} \in \operatorname{Inv}^{\bullet}\left(\mu_{2}^{n}, \mathrm{~K}_{2}\right)$.

Proof. The fact that all normalised invariants are of 2-torsion is an obvious consequence of the inclusion $\operatorname{Inv}^{\bullet}\left(\mathrm{BO}_{\mathrm{n}}, \mathrm{M}\right) \subset \operatorname{Inv}^{\bullet}\left(\mathrm{B} \mu_{2}^{n}, \mathrm{M}\right)$. Note that moreover, we have $\operatorname{Inv}^{\bullet}\left(\mathrm{BO}_{n}, \mathrm{M}\right) \subset \operatorname{Inv} \bullet\left(\mathrm{B} \mu_{2}^{n}, \mathrm{M}\right)^{\mathrm{S}_{\mathrm{n}}}$, by the gluing conditions, and $\operatorname{Inv}^{\bullet}\left(\mathrm{B} \mu_{2}^{n}, \mathrm{M}\right)^{\mathrm{S}_{\mathrm{n}}}$ is exactly the group in our formula. The description of the cohomological invariants with coefficients in a $\mathrm{K}_{2}$ is obtained in [15, Sec. 23]; the description for an arbitrary cycle module then follows by Lemma 5.4 as $S_{n}$ acts on $\mu_{2}^{n}$ by permuting the components.

Remark 5.6. In [17, Sec. 6], Guillot claims that there are no Stiefel-Whitney classes with coefficients in $\mathrm{K}_{\mathrm{Mil}}$, but he is only computing the degree 1 component. An example of such a class in degree 2 is

$$
\alpha_{1}\{-1\} \in \operatorname{Inv}^{2}\left(\mathrm{BO}_{n}, \mathrm{~K}_{\mathrm{Mil}}\right)
$$

when $k$ does not contain a square root of -1 .

Theorem 5.7 (Gille and Hirsch). Let $(V, b)$ be a finite-dimensional, regular symmetric bilinear space over $k$ and let $W \subset \mathrm{O}(V, b)$ be a finite subgroup. Assume that $S(V)^{W}$ is a polynomial ring over $k$. Let $G_{1}, \ldots, G_{r}$ be a set of representatives, up to conjugation, of the maximal abelian subgroups of $W$ that are generated by reflections. Then the pullback

$$
\operatorname{Inv}^{\bullet}(\mathrm{B}(W), \mathrm{M}) \rightarrow \prod_{i} \operatorname{Inv}^{\bullet}\left(\mathrm{B}\left(G_{i}\right), \mathrm{M}\right)^{N_{W}\left(G_{i}\right)}
$$

is injective.

Proof. This is a combination of [16, Thm. 7, Cor. 8].

Remark 5.8. Gille and Hirsch additionally prove the splitting principle for invariants with coefficients in Witt groups, which do not form a cycle module but have strong enough properties that the (classical) theory of cohomological invariants still works.

Corollary 5.9. Let $W$ be as before. Than any normalised cohomological invariant of $\mathrm{B} W$ is of 2-torsion - that is,

$$
2 \cdot\left(\operatorname{Inv}{ }^{\bullet}(\mathrm{B}(W), \mathrm{M}) / \mathrm{M}^{\bullet}(k)\right)=0 .
$$

Proof. This is immediate from Lemma 5.3.

We now extend the computations of the invariants of some classical groups to arbitrary cycle modules.

Proposition 5.10. We have the following:

1. $\operatorname{Inv}^{\bullet}\left(\mathrm{BS}_{n}, \mathbf{M}\right)=\mathbf{M}^{\bullet}(k) \oplus \alpha_{1} \cdot \mathbf{M}^{\bullet}(k)_{2} \oplus \cdots \oplus \alpha_{[n / 2]} \cdot \mathbf{M}^{\bullet}(k)_{2}$.

2. When $n$ is odd, $\operatorname{Inv}^{\bullet}\left(\mathrm{BSO}_{n}, \mathrm{M}\right)=\mathrm{M}^{\bullet}(k) \oplus_{2 j \leq n} \alpha_{2 j} \cdot \mathrm{M}^{\bullet}(k)_{2}$.

3. $\operatorname{Inv}^{\bullet}\left(\mathrm{BPGL}_{2}, \mathbf{M}\right)=\mathbf{M}^{\bullet}(k) \oplus w_{2} \cdot \mathbf{M}^{\bullet}(k)_{2}$.

In particular, all normalised invariants are of 2-torsion.

Proof. We start by proving (1). Let $H \simeq(\mathbb{Z} / 2 \mathbb{Z})^{\times m}$ be the subgroup of $S_{\mathrm{n}}$ generated by the transpositions $(1,2),(3,4), \ldots,(n-1, n)$ for $n$ even and by $(1,2), \ldots,(n-2, n-1)$ for $n$ odd. Observe that if $m=\lfloor n / 2\rfloor$, the normaliser of $H$ is a copy of $\mathrm{S}_{\mathrm{m}}$, which acts on $H$ by permuting its generators. By Theorem 5.7, the pullback of invariants along the morphism $\mathrm{BH} \rightarrow \mathrm{BS}_{n}$ induces an injective morphism

$$
\operatorname{Inv}^{\bullet}\left(\mathrm{BS}_{n}, \mathrm{M}\right) \longrightarrow \operatorname{Inv}^{\bullet}(\mathrm{B} H, \mathrm{M})^{\mathrm{S}_{\mathrm{m}}}
$$


for all M. Then by Lemma 5.4 we only need to prove the case of $M=K_{2}$. This is done in in [15, Thm. 25.6.(1)].

To prove (2), note that for $n$ odd, we have $\mathrm{SO}_{n}=\mathrm{O}_{\mathrm{n}} \times \mu_{2}$. Then we can combine Proposition 4.3 with Proposition 5.5 to conclude that

$$
\operatorname{Inv}^{\bullet}\left(\mathrm{BSO}_{n}, \mathrm{M}\right)=\operatorname{Inv}^{\bullet}\left(\mathrm{BO}_{n}, \mathrm{M}\right) / \alpha_{1} \cdot \operatorname{Inv}^{\bullet}\left(\mathrm{BO}_{n}, \mathrm{M}\right),
$$

where the multiplication is given by seeing $\alpha_{1}$ as an element of $\operatorname{Inv}^{\bullet}\left(\mathrm{BO}_{\mathrm{n}}, \mathrm{K}_{2}\right)$. The multiplicative structure of $\operatorname{Inv}^{\bullet}\left(\mathrm{BO}_{\mathrm{n}}, \mathrm{K}_{2}\right)$ (see [15, Remark 17.4]) immediately shows that the resulting submodule is generated by the odd-degree Stiefel-Whitney classes. Our claim follows.

To prove the last statement, we just note that in characteristic different from 2 we have an isomorphism $\mathrm{PGL}_{2} \simeq \mathrm{SO}_{3}$.

When $n$ is even, computing the cohomological invariants of $\mathrm{BSO}_{n}$ is more complicated. The case $\mathbf{M}=\mathrm{H}_{\mathbb{Z} / \ell \mathbb{Z}}$ is done in [15, Sec. 20], as well as in [17, Prop 3.4.1] with methods that are closer to ours. While it is probably not too hard to extend the computation to arbitrary cycle modules, it would be beyond the scope of this paper.

As an application of Proposition 5.10, we compute the Brauer groups of $\mathrm{BS}_{n}$ and $\mathrm{BPGL}_{2}$ :

Corollary 5.11. We have

$$
{ }^{c} \operatorname{Br}\left(\mathrm{BS}_{n}\right)={ }^{c} \operatorname{Br}(k) \oplus \mathrm{H}^{1}(k, \mathbb{Z} / 2 \mathbb{Z}) \oplus \mathbb{Z} / 2 \mathbb{Z}, \quad{ }^{c} \operatorname{Br}\left(\mathrm{BPGL}_{2}\right)={ }^{c} \operatorname{Br}(k) \oplus \mathbb{Z} / 2 \mathbb{Z} .
$$

Proof. The formulas for the cohomological Brauer group are immediate from the description of the generalised cohomological invariants given in Proposition 5.10.

For $\mathrm{BS}_{n}$, we know that the Brauer group is equal to the cohomological Brauer group thanks to Proposition 2.2.

For $\mathrm{BPGL}_{2}$, note that the universal conic $\mathcal{C}=\left[\mathrm{P}^{1} / \mathrm{PGL}_{2}\right]$ induces a nonzero element $\{\mathcal{C}\}$ in the Brauer group which is trivial when pulled back to the base field. This implies that $\{\mathcal{C}\}=w_{2}+\alpha_{0}$, where $\alpha_{0}$ belongs to $\operatorname{Br}(k)$, showing that $\mathrm{Br}\left(\mathrm{BPGL}_{2}\right)=\mathrm{Br}^{\prime}\left(\mathrm{BPGL}_{2}\right)$.

\section{The moduli stacks of hyperelliptic curves}

We briefly review Arsie and Vistoli's presentation of the moduli stack $\mathcal{H}_{g}$, and recall some results from [8] and that will be needed later. Let $n$ be an even positive integer, and consider the affine space $\mathbb{A}^{n+1}$, seen as the space of binary forms of degree $n$. There are two different natural actions on this space:

$\circ$ an action of $\mathrm{GL}_{2}$ given by

$$
A \cdot f\left(x_{0}, x_{1}\right)=\operatorname{det}(A)^{n / 2-1} f\left(A^{-1}\left(x_{0}, x_{1}\right)\right)
$$

$\circ$ an action of $\mathrm{PGL}_{2} \times \mathbb{G}_{m}$ given by

$$
([A], t) \cdot f\left(x_{0}, x_{1}\right)=\operatorname{det}(A)^{n / 2} t^{-2}\left(f\left(A^{-1}\left(x_{0}, x_{1}\right)\right)\right) .
$$

The open subset of square-free forms inside $\mathbb{A}^{n+1}$ is $G$-invariant. We will denote it by $\mathbb{A}_{\mathrm{sm}}^{n+1}$.

Theorem 6.1. When $g \geq 2$ is even, we have an isomorphism $\left[\mathbb{A}_{\mathrm{sm}}^{2 g+3} / \mathrm{GL}_{2}\right] \simeq \mathcal{H}_{g}$.

When $g \geq 3$ is odd, we have an isomorphism $\left[\mathbb{A}_{\mathrm{sm}}^{2 g+3} /\left(\mathrm{PGL}_{2} \times \mathbb{G}_{m}\right)\right] \simeq \mathcal{H}_{g}$.

Proof. This is proven in $[2,4.7]$. 
When no confusion is possible, we will write $G$ for either $\mathrm{GL}_{2}$ or $\mathrm{PGL}_{2} \times \mathbb{G}_{m}$. Our computation will be for the most part done on the projectivisations

$$
P^{n}=\left(\mathbb{A}^{n+1} \backslash\{0\}\right) / \mathbb{G}_{m}
$$

with the induced action of $G$.

The following $G$-invariant stratification will be crucial. Let $\Delta_{i}^{n} \subset P^{n}$ be the closed subscheme of $P^{n}$ whose points are forms of degree $n$ which are divisible by the square of a form of degree at least $i$, with the reduced subscheme structure. Then

$$
P^{n} \supset \Delta_{1}^{n} \supset \cdots \supset \Delta_{n / 2}^{n}
$$

is a $G$-invariant stratification of $P^{n}$.

We define $\Delta_{[i]}^{n}$ as the subscheme of forms of degree $n$ divisible by the square of a form of degree $i$ but not higher - that is, $\Delta_{[i]}^{n}=\Delta_{i}^{n} \backslash \Delta_{i+1}^{n}$ - and similarly, given $j>i$ we define $\Delta_{[i, j]}^{n}=\Delta_{i}^{n} \backslash \Delta_{j+1}^{n}$. Finally, in keeping with this notation, we define the open subsets $P_{\mathrm{sm}}^{n}=P_{[0]}^{n}=P^{n} \backslash \Delta_{1}^{n}$ and $P_{[0, i]}^{n}=P^{n} \backslash \Delta_{i+1}^{n}$.

There is a natural map $P^{n-2 r} \times P^{r} \rightarrow \Delta_{r}^{n}$ given by $(f, g) \rightarrow f g^{2}$. Checking that the map is equivariant with respect to the action of $G$ is easy. Note that if we restrict the map to $P_{\mathrm{sm}}^{n-2 r} \times P^{r}$, the image is exactly $\Delta_{[r]}^{n}$.

Proposition 6.2. The map $P_{\mathrm{sm}}^{n-2 r} \times P^{r} \rightarrow \Delta_{[r]}^{n}$ is an equivariant universal homeomorphism.

Proof. This is [21, Prop. 3.3].

Consider the map $\left(P^{1}\right)^{n} \rightarrow P^{n}$ given by $\left(l_{1}, \ldots, l_{n}\right) \mapsto l_{1} \cdots l_{n}$. If we restrict the map to $P_{\mathrm{sm}}^{n}$, it becomes an $\mathrm{S}_{\mathrm{n}}$-torsor, thus inducing a map $P_{\mathrm{sm}}^{n} \rightarrow \mathrm{BS}_{n}$.

Another way of seeing this is the following: consider the stack ét $t_{n}$ of étale algebras of degree $n$. There is a natural equivalence ét ${ }_{n} \simeq \mathrm{BS}_{n}$. Given a point $f: S \rightarrow P_{\mathrm{sm}}^{n}$, we get an étale algebra by taking the zero locus of the form $f$ over $P_{S}^{1}$. One can easily check that these two maps coincide.

Now, the stack $\left[P_{\mathrm{sm}}^{n} / G\right]$ parametrises families of conics $C^{\prime} / S$ (trivial families if $G=\mathrm{GL}_{2}$ ) equipped with a line bundle $L$ of vertical degree $n / 2$ and a subscheme $W_{C^{\prime}}$ of codimension 1, étale on the base, whose associated divisor is in the linear series of $L^{\otimes 2}$.

Taking the subscheme $W_{C^{\prime}} / S$, we get a map to ét ${ }_{n}=\mathrm{BS}_{n}$. It is easy to check that this map provides a factorisation to the maps alread mentioned, and that moreover it extends to $\mathbb{A}_{\mathrm{sm}}^{n+1}$ and $\left[\mathbb{A}_{\mathrm{sm}}^{n+1} / G\right]$. When we take the stack $\mathcal{H}_{g}$, this map is precisely the map that sends a hyperelliptic curve over $S$ to its Weierstrass divisor, seen as an $S$-scheme.

We want to show that the map $\mathbb{A}_{\mathrm{sm}}^{n+1} \rightarrow \mathrm{BS}_{n}$ is smooth Nisnevich. This in turn will imply that given a smooth stack $\mathcal{X}$ and a map $f: \mathcal{X} \rightarrow \mathrm{BS}_{n}$ with a factorisation

$$
\mathbb{A}_{\mathrm{sm}}^{n+1} \rightarrow \mathcal{X} \rightarrow \mathrm{BS}_{n}
$$

the map $f$ is smooth Nisnevich as well. To do so, it suffices to show that the image contains a versal torsor. Write a form $f=a_{0} x_{0}^{n}+\cdots+a_{n} x_{1}^{n}$. The restriction of the map $P_{\mathrm{sm}}^{n} \rightarrow \mathrm{BS}_{n}$ to the complement of the hyperplane $a_{0}=0$ comes from the generically free $S_{n}$-representation on $\mathbb{A}^{n}$ given by permuting the coordinates. As the torsor induced by the generic point of a generically free representation is always versal, we conclude that the map $\mathbb{A}_{\mathrm{sm}}^{n+1} \rightarrow \mathrm{BS}_{n}$ is smooth Nisnevich. This immediately implies the following:

Proposition 6.3. Let $n$ be an even positive integer. The pullback map $\operatorname{Inv}^{\bullet}\left(\mathrm{BS}_{n}, \mathrm{M}\right) \rightarrow \operatorname{Inv}^{\bullet}(\mathcal{X}, \mathrm{M})$, where $\mathcal{X}$ is either $\mathbb{A}_{\mathrm{sm}}^{n+1},\left[\mathbb{A}_{\mathrm{sm}}^{n+1} / G\right]$ or $\left[P_{\mathrm{sm}}^{n} / G\right]$, is injective.

Proof. This is a combination of [8, Prop. 3.1- Cor. 3.2]. 


\section{The generalised cohomological invariants of $\mathcal{H}_{g}$}

We are ready to compute the cohomological invariants of $\mathcal{H}_{g}$ with coefficients in an arbitrary $\ell$-torsion cycle module $M$. The specialisation of this computation to degree 2 invariants with coefficients in $\mathrm{H}_{\mu_{\ell}^{\vee}}$ will give us the result on the Brauer groups.

From now on, the cycle module $\mathrm{M}$ will be always assumed to be $\ell$-torsion.

It is worth noting that computing only the degree 2 invariants does not seem any easier than computing (almost) the full group in this case (the highest-degree generator needs some extra effort, as one can see from the proof of Theorem 7.9 and Remark 7.10), but in more general situations it might be possible to obtain the result in low degrees even if the higher degrees seem too hard to approach.

First we need a few more lemmas from [21,23]. Recall that

$$
\mathrm{CH}_{\mathrm{GL}_{2}}^{*}\left(P^{n}\right)=\mathbb{Z}\left[t, \lambda_{1}, \lambda_{2}\right] /\left(R_{n}\left(t, \lambda_{1}, \lambda_{2}\right)\right),
$$

where $\lambda_{1}, \lambda_{2}$ are the Chern classes of the standard representation of $\mathrm{GL}_{2}, t$ is the first Chern class of $\mathcal{O}_{P^{n}}(-1)$ and $R_{n}\left(t, \lambda_{1}, \lambda_{2}\right)$ is a polynomial of degree $n$. We also have

$$
\mathrm{CH}_{\mathrm{PGL}_{2} \times \mathbb{G}_{m}}^{*}\left(P^{n}\right)=\mathbb{Z}\left[t, s, c_{2}, c_{3}\right] /\left(T_{n}\left(t, c_{2}, c_{3}\right), 2 c_{3}\right),
$$

where $t$ is the first Chern class of $\mathcal{O}_{P^{n}}(-1), s$ is the first Chern class of the standard representation of $\mathbb{G}_{m}$ and $c_{2}, c_{3}$ are the second and third Chern classes of the 3-dimensional representation of PGL coming from the isomorphism $\mathrm{PGL}_{2} \simeq \mathrm{SO}_{3}$.

We should also note that if $\mathbb{G}_{m}$ acts trivially on $X$, then $A_{\mathbb{G}_{m}}^{*}(X, \mathrm{M})=A^{*}(X, \mathrm{M}) \otimes \mathbb{Z}[s]$. In particular, for our computations we can often consider $G=\mathrm{PGL}_{2}$ rather than $\mathrm{PGL}_{2} \times \mathbb{G}_{m}$.

Lemma 7.1. The class of $\Delta_{1}^{n}$ in $\mathrm{CH}_{\mathrm{GL}_{2}}^{1}\left(P^{n}\right)$ is $2(n-1)\left(t+n \lambda_{1}\right)$. The class of $\Delta_{1}^{n}$ in $\mathrm{CH}_{\mathrm{PGL}_{2}}^{1}\left(P^{n}\right)$ is $2(n-1) t$.

Proof. For $G=\mathrm{GL}_{2}$, the statement is proved in [21, Prop. 4.3]. For $G=\mathrm{PGL}_{2}$, see [5, Prop. 5.2], albeit there is a mistake in the statement of the result, where $4(n-2) h_{n}$ should be replaced by $(4 n-2) h_{n}$ (note that the $n$ in the statement of [5, Prop. 5.2] would be $n / 2$ in our lemma's notation).

We begin by dealing with the case of odd torsion, which is easy due to the following lemmas.

Lemma 7.2. Consider the natural action of $\mathrm{PGL}_{2}$ on $P^{1}$. We have

$$
A_{\mathrm{PGL}_{2}}^{*}\left(P^{1}, \mathrm{M}\right) \simeq A_{\mathbb{G}_{m}}^{*}(\operatorname{Spec}(k), \mathrm{M})=\mathbb{Z}[t] \otimes \mathrm{M}^{\bullet}(k) .
$$

Proof. We have

$$
A_{G}^{*}\left(P^{1}, \mathrm{M}\right) \simeq A_{H}^{*}(\operatorname{Spec}(k), \mathrm{M})
$$

where $H$ is the stabiliser of a point in $P^{1}$, which is isomorphic to a semidirect product $\mathbb{G}_{m} \ltimes \mathbb{G}_{a}$. Then by $[23,8]$ we have

$$
A_{H}^{*}(\operatorname{Spec}(k), \mathrm{M}) \simeq A_{\mathbb{G}_{m}}^{*}(\operatorname{Spec}(k), \mathrm{M}),
$$

which allows us to conclude immediately.

Lemma 7.3. Let $\ell$ be odd. Then the pullback map $A_{G}^{0}\left(P_{\mathrm{sm}}^{n}, \mathrm{M}\right) \rightarrow A_{G}^{0}\left(P_{\mathrm{sm}}^{n} \times P^{1} \times P^{1}, \mathrm{M}\right)$ is surjective.

We postpone the proof this lemma, as it involves an argument similar to the one used in the next lemma and in the proposition after it.

Lemma 7.4. Suppose $\ell=p^{n}$, with $p$ a prime number different from 2. Then $A_{G}^{0}\left(\Delta_{1}^{n}, \mathrm{M}\right)=\mathbf{M}^{\bullet}(k)$. 
Proof. As $A_{G}^{0}\left(\Delta_{1}^{n}, \mathrm{M}\right)$ is isomorphic to $A_{G}^{0}\left(\Delta_{[1,2]}^{n}, \mathrm{M}\right)$ (because $\Delta_{3}^{n}$ has codimension 2 in $\left.\Delta_{1}^{n}\right)$, we can compute it using the following exact sequence:

$$
0 \rightarrow A_{G}^{0}\left(\Delta_{[1,2]}^{n}, \mathrm{M}\right) \rightarrow A_{G}^{0}\left(\Delta_{[1]}^{n}, \mathrm{M}\right) \stackrel{\partial}{\rightarrow} A_{G}^{0}\left(\Delta_{[2]}^{n}, \mathrm{M}\right)
$$

We want to prove that the kernel of $\partial$ is equal to $\mathbf{M}^{\bullet}(k)$. This will then imply that the image of $A_{G}^{0}\left(\Delta_{[1,2]}^{n}, \mathrm{M}\right)$ must be equal to $\mathrm{M}^{\bullet}(k)$, thus showing that $A_{G}^{0}\left(\Delta_{[1,2]}^{n}, \mathrm{M}\right)=\mathrm{M}^{\bullet}(k)$.

The map $P_{[0,1]}^{n-2} \times P^{1} \stackrel{\pi}{\rightarrow} \Delta_{[1,2]}^{n}$ yields the following commutative diagram with exact columns:

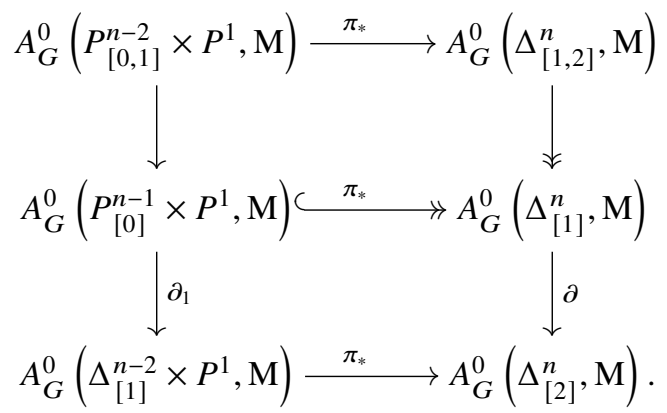

The second horizontal map is an isomorphism by Proposition 2.23, because $\pi$ is a universal homeomorphism when restricted to $\Delta_{[1]}^{n}$ (Proposition 6.2).

The kernel of $\partial_{1}$ is $\mathrm{M}^{\bullet}(k)$, because $A_{G}^{0}\left(P_{[0,1]}^{n-2} \times P^{1}, \mathrm{M}\right)=\mathrm{M}^{\bullet}(k)$, as we have $A_{G}^{0}\left(P^{n-2} \times P^{1}, \mathrm{M}\right)=$ $\mathrm{M}^{\bullet}(k)$ by the projective bundle formula and $\Delta_{2}^{n-2} \times P^{r}$ has codimension 2 .

We claim that the third horizontal map is injective, implying that the kernel of $\partial$ must be equal to $\mathbf{M}^{\bullet}(k)$ as well.

Let $\psi$ be the map from $P_{[0]}^{n-4} \times P^{1} \times P^{1}$ to $P_{[0]}^{n-4} \times P^{2}$ sending $(f, g, h)$ to $(f, g h)$. We have a commutative diagram:

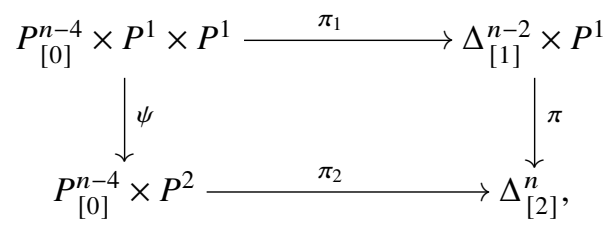

where $\pi_{1}$ and $\pi_{2}$ are defined respectively by $(f, g, h) \rightarrow\left(f g^{2}, h\right)$ and $(f, g) \rightarrow\left(f g^{2}\right)$. The maps $\pi_{1}$ and $\pi_{2}$ are universal homeomorphisms, so the push-forward maps $\left(\pi_{1}\right)_{*},\left(\pi_{2}\right)_{*}$ are isomorphisms. Then if we prove that $\psi_{*}$ is injective, $\pi_{*}$ will be injective too. Consider this last diagram:

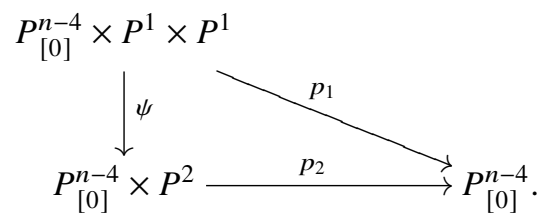

The pullbacks along $p_{1}$ and $p_{2}$ are both surjective, the first due to Lemma 7.3 and the second due to the projection formula, implying that the pullback along $\psi$ is surjective. We have $\psi_{*}\left(\psi^{*} \alpha\right)=\operatorname{deg}(\psi) \alpha$ by the projection formula. Then, as the degree of $\psi$ is $2, \psi_{*}$ is injective. 
The Lemma 7.4 tells us that when $\mathrm{M}$ is of odd torsion, the group $A_{G}^{0}\left(P_{\mathrm{sm}}^{n}, \mathrm{M}\right)$ must fit in the following exact sequence:

$$
0 \rightarrow \mathrm{M}^{\bullet}(k)=A_{G}^{0}\left(P^{n}, \mathrm{M}\right) \rightarrow A_{G}^{0}\left(P_{\mathrm{sm}}^{n}, \mathrm{M}\right) \rightarrow \mathrm{M}^{\bullet}(k)=A_{G}^{0}\left(\Delta_{1}^{n}, \mathrm{M}\right) \rightarrow A_{G}^{1}\left(P^{n}, \mathrm{M}\right)
$$

So, roughly speaking, it can contain, other than the submodule $\mathbf{M}^{\bullet}(k)$ of constant invariants, at most an additional copy of the invariants of the base field, shifted in degree by 1 . This is of course imprecise at this point, as the sequence may not split.

In the next proposition we compute the kernel of the last map, and moreover we will show that the group $A_{G}^{0}\left(P_{\mathrm{sm}}^{n}, \mathrm{M}\right)$ splits as a direct sum of a copy of the elements coming from $A_{G}^{0}\left(P^{n}, \mathrm{M}\right)$ and the kernel. This will allow us to easily compute the cohomological invariants of $\mathcal{H}_{g}$ with coefficients in $\mathrm{M}$.

Proposition 7.5. Assume $\ell$ is odd. Let $\ell^{\prime}$ be the greatest common divisor of $\ell$ and $2 g+1$. Then

$$
\operatorname{Inv}^{\bullet}\left(\mathcal{H}_{g}, \mathbf{M}\right) \simeq \mathbf{M}^{\bullet}(k) \oplus \mathbf{M}^{\bullet}(k)_{\ell^{\prime}}[1] .
$$

In particular, if $\ell$ is coprime with $2 g+1$, the normalised cohomological invariants of $\mathcal{H}_{g}$ with coefficients in $\mathrm{M}$ are trivial.

Proof. We begin by computing the invariants of $\left[P_{\mathrm{sm}}^{2 g+2} / G\right]$.

First consider the case of $\mathrm{M}=\mathrm{K}_{\ell}$. Consider the exact sequence

$$
\begin{gathered}
0 \longrightarrow A_{G}^{0}\left(P^{2 g+2}, \mathbb{Z} / p^{n} \mathbb{Z}\right) \longrightarrow A_{G}^{0}\left(P_{\mathrm{sm}}^{2 g+2}, \mathbb{Z} / p^{n} \mathbb{Z}\right) \\
\rightarrow A_{G}^{0}\left(\Delta_{1}^{2 g+2}, \mathbb{Z} / p^{n} \mathbb{Z}\right) \longrightarrow A_{G}^{1}\left(P^{2 g+2}, \mathbb{Z} / p^{n} \mathbb{Z}\right) \longrightarrow A_{G}^{1}\left(P_{\mathrm{sm}}^{2 g+2}, \mathbb{Z} / p^{n} \mathbb{Z}\right) .
\end{gathered}
$$

We have $A_{G}^{0}\left(\Delta_{1}^{2 g+2}, \mathrm{~K}_{\ell}\right)=\mathrm{K}_{\ell}^{\bullet}(k)$, and the push-forward map is just multiplication by $\left\{\Delta_{1}^{2 g+2}\right\}$. Assume first that $\ell$ divides $2 g+1$, so that the image of $\left\{\Delta_{1}^{2 g+2}\right\}$ is zero. Then if we pick an inverse image $\gamma$ of 1 , the submodule $\gamma \cdot \mathrm{K}_{\ell}^{\bullet}(k)$ maps isomorphically to $A_{G}^{0}\left(\Delta_{1, n}, \mathrm{~K}_{\ell}\right)$, splitting the exact sequence.

Now let $\ell$ be arbitrary and let $\ell^{\prime}$ be the greatest common divisor of $\ell$ and $2 g+1$. Let $\gamma \in$ $A_{G}^{0}\left(P_{\mathrm{sm}}^{2 g+2}, \mathrm{~K}_{\ell^{\prime}}\right)$ be as before.

Then for any $x \in \mathrm{K}_{\ell}^{\bullet}(k)_{\ell^{\prime}}$, the element $\gamma \cdot x$ belongs to $A^{0}\left(P_{\mathrm{sm}}^{2 g+2}, \mathrm{~K}_{\ell}\right)$, and moreover the boundary map sends $\gamma \cdot \mathrm{K}_{\ell}^{\bullet}(k)_{\ell^{\prime}}$ to $\mathrm{K}_{\ell}^{\bullet}(k)_{\ell^{\prime}} \subset A^{0}\left(\Delta_{1}^{2 g+2}, \mathrm{~K}_{\ell}\right)$, which is exactly the kernel, splitting the exact sequence.

For an arbitrary $\ell$-torsion cycle module $\mathbf{M}$, take $\gamma$ as before. We have an inclusion $\gamma \cdot \mathbf{M}^{\bullet}(k)_{\ell^{\prime}} \subseteq$ $A_{G}^{0}\left(P_{\mathrm{sm}}^{2 g+2}, \mathrm{M}\right)$, as these elements are clearly unramified. On the other hand, using the fact that $A_{G}^{0}\left(\Delta_{1}^{2 g+2}, \mathrm{~K}_{\ell}\right)=\mathrm{M}^{\bullet}(k)$, it is easy to see that we must have $A_{G}^{0}\left(P_{\mathrm{sm}}^{2 g+2}, \mathrm{M}\right)=\gamma \cdot \mathrm{M}^{\bullet}(k)_{\ell^{\prime}}$.

The next step consists of verifying that the $\mathbb{G}_{m}$-torsor $\mathcal{H}_{g} \rightarrow\left[P_{\mathrm{sm}}^{2 g+2} / G\right]$ does not generate any new invariant. This can be done as in the proof of Theorem 7.9. It is equivalent to proving that the first Chern class of the associated line bundle $\mathcal{L}$ does not annihilate any element of $A_{G}^{0}\left(P_{\mathrm{sm}}^{2 g+2}, \mathrm{M}\right)$; as the class is injective on the copy of $\mathrm{M}^{\bullet}(k)$ coming from the base field, we only have to check it on the submodule $\gamma \cdot \mathrm{M}^{\bullet}(k)$. This is done exactly as in the proof of Theorem 7.9 , by taking the boundary of the elements and verifying that it is nonzero.

Proof of Lemma 7.3. First note that if $G$ is $\mathrm{GL}_{2}$ or in the nonequivariant case, the statement is obvious by the projective bundle formula. 
Now consider the case where $G=\mathrm{PGL}_{2}$. We have

$$
A_{G}^{0}\left(P_{\mathrm{sm}}^{n} \times P^{1} \times P^{1}, \mathrm{M}\right) \simeq A_{H}^{0}\left(P_{\mathrm{sm}}^{n} \times P^{1}, \mathrm{M}\right)
$$

where $H$ is the stabiliser of a point in $P^{1}$, which is isomorphic to a semidirect product $\mathbb{G}_{m} \ltimes \mathbb{G}_{a}$. As $H$ is a special group, the pullback

$$
A_{H}^{0}\left(P_{\mathrm{sm}}^{n} \times P^{1}, \mathrm{M}\right) \rightarrow A^{0}\left(P_{\mathrm{sm}}^{n} \times P^{1}, \mathrm{M}\right)=A^{0}\left(P_{\mathrm{sm}}^{n}, \mathrm{M}\right)
$$

has to be injective. We claim that the pullback $A_{G}^{0}\left(P_{\mathrm{sm}}^{n}, \mathrm{M}\right) \rightarrow A^{0}\left(P_{\mathrm{sm}}^{n}, \mathrm{M}\right)$ is surjective, which would allow us to conclude immediately.

One can use the same techniques used to prove Lemma 7.4 to easily show that when $p \neq 2$, the nonequivariant group $A^{0}\left(\Delta_{1}^{n}, \mathrm{M}\right)$ is equal to $\mathrm{M}^{\bullet}(k)$, and thus $A^{0}\left(P_{\mathrm{sm}}^{n}, \mathrm{M}\right)$ either is $\mathrm{M}^{\bullet}(k)$ or is generated by 1 and an element in degree 1 corresponding to the equation for $\Delta_{1}^{n}$, multiplied by the submodule of $\mathbf{M}^{\bullet}(k)$ which annihilates the class of $\Delta_{1}^{n}$ in $A^{1}\left(P^{n}, \mathrm{M}\right)$.

In the latter case, consider the following commutative diagram induced by the pullback from equivariant to nonequivariant Chow groups with coefficients:

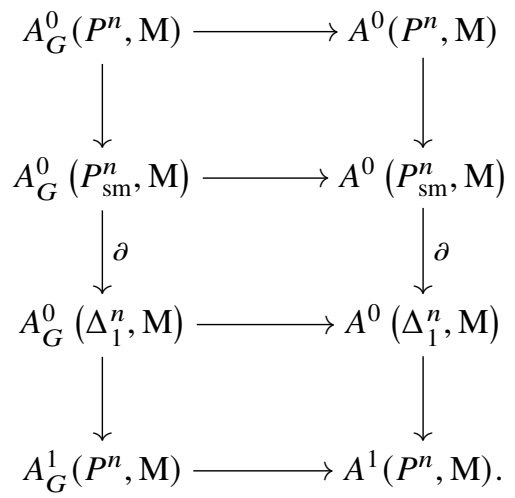

Both the top and the bottom horizontal map are isomorphisms, as one can see using the fact that the groups on the top row are $M(k)$ and the groups on the bottom row are given by $c_{1}^{G}\left(\mathcal{O}_{P^{n}}(-1)\right) \cdot \mathrm{M}^{\bullet}(k)$.

Moreover, $A^{0}\left(P_{\mathrm{sm}}^{n}, \mathrm{M}\right)$ is generated as by $1 \cdot \mathbf{M}^{\bullet}(k)$ and an element $\alpha \cdot \mathbf{M}^{\bullet}(k)$, where $\alpha$ is an element such that $\partial(\alpha)=1$ in $A_{G}^{0}\left(\Delta_{1}^{n}, \mathrm{M}\right)$, with no additional relations.

The third horizontal map maps $1 \in A_{G}^{0}\left(\Delta_{1}^{n}, \mathrm{M}\right)$ to $1 \in A^{0}\left(\Delta_{1}^{n}, \mathrm{M}\right)$, which shows that an element $\tau$ of degree 0 maps to 0 in the equivariant group $A_{G}^{1}\left(P^{n}, \mathrm{M}\right)$ if and only if it maps to 0 in $A^{1}\left(P^{n}, \mathrm{M}\right)$. Then there must be an element

$$
\alpha^{\prime} \in A_{G}^{0}\left(P_{\mathrm{sm}}^{n}, \mathrm{M}\right)
$$

which maps to $\alpha \in A^{0}\left(P_{\mathrm{sm}}^{n}, \mathrm{M}\right)$, and thus the pullback $A_{G}^{0}\left(P_{\mathrm{sm}}^{n}, \mathrm{M}\right) \rightarrow A^{0}\left(P_{\mathrm{sm}}^{n}, \mathrm{M}\right)$ is surjective. This concludes the proof.

The even case is much more complicated. We first compute the invariants with coefficients in $\mathrm{K}_{2^{r}}$ of $\left[P_{\mathrm{sm}}^{2 g+2} / G\right]$. Our computation is based on the fact that we already know that the cohomological invariants of $\mathrm{S}_{\mathrm{n}}$ inject into those of $\left[P_{\mathrm{sm}}^{2 g+2} / G\right]$. Using this, we inductively show that 'there is no more room' and we have found all the invariants. This is done for $r=1$ in [8]. 
Lemma 7.6. We have the following:

- If $g$ is even, then

$$
\mathrm{Inv}^{\bullet}\left(\left[P_{\mathrm{sm}}^{2 g+2} / G\right], \mathrm{K}_{2^{r}}\right) \simeq \mathrm{Inv}^{\bullet}\left(\mathrm{BS}_{2 g+2}, \mathrm{~K}_{2^{r}}\right)
$$

○ If $g$ is odd, then

$$
\mathrm{Inv}^{\bullet}\left(\left[P_{\mathrm{sm}}^{2 g+2} / G\right], \mathrm{K}_{2^{r}}\right) \simeq \mathrm{Inv}^{\bullet}\left(\mathrm{BS}_{2 g+2}, \mathrm{~K}_{2^{r}}\right) \oplus \mathrm{K}_{2^{r}}^{\bullet}(k)_{2}[2],
$$

where the copy of $\mathrm{K}_{2^{r}}^{\bullet}(k)_{2}$ [2] comes from the cohomological invariants of $\mathrm{PGL}_{2}$.

Proof. Consider the exact sequence

$$
A_{G}^{0}\left(P^{n}, \mathrm{~K}_{2^{r}}\right) \hookrightarrow A_{G}^{0}\left(P_{\mathrm{sm}}^{n}, \mathrm{~K}_{2^{r}}\right) \stackrel{\partial}{\rightarrow} A_{G}^{0}\left(\Delta_{1}^{n}, \mathrm{~K}_{2^{r}}\right) \subset A_{G}^{0}\left(P_{\mathrm{sm}}^{n-2} \times P^{1}, \mathrm{~K}_{2^{r}}\right) .
$$

First consider the case $r=1$. We will proceed by induction on $n$. If we assume the result for $n-2$, we have that $A_{G}^{0}\left(P_{\mathrm{sm}}^{n-2} \times P^{1}, \mathrm{~K}_{2} r\right)=\operatorname{Inv}^{\bullet}\left(\mathrm{BS}_{n-2}, \mathrm{~K}_{2}\right)$. Note that if $G=\mathrm{PGL}_{2}$, the invariant $w_{2}$ in $A_{G}^{0}\left(P_{\mathrm{sm}}^{n-2} \times P^{1}, \mathrm{~K}_{2^{r}}\right)$ is killed by the $P^{1}$. The cokernel of the first is surjective on the normalised invariants of $S_{\mathrm{n}}$. Thus we have a map

$$
\operatorname{Inv}^{\bullet}\left(\mathrm{BS}_{n}, \mathrm{~K}_{2}\right) / \mathrm{K}_{2}^{\bullet}(k) \rightarrow \operatorname{Inv}^{\bullet}\left(\mathrm{BS}_{n-2}, \mathrm{~K}_{2}\right)_{2}
$$

which lowers degree by 1 . Comparing the generators of the image with the generators of $A_{G}^{0}\left(P_{\mathrm{sm}}^{n-2} \times P^{1}, \mathrm{~K}_{2^{r}}\right)$ degree by degree shows that the map is surjective, and in particular there cannot be any additional element in $A_{G}^{0}\left(P_{\mathrm{sm}}^{n}, \mathrm{~K}_{2}\right)$, yielding the result.

Now pick $r>1$. We will proceed by induction on $n$ even. If we assume the result for $n-2$, we have that $A_{G}^{0}\left(P_{\mathrm{sm}}^{n-2} \times P^{1}, \mathrm{~K}_{2^{r}}\right)=\operatorname{Inv}^{\bullet}\left(\mathrm{BS}_{n-2}\right)$. Note that if $G=\mathrm{PGL}_{2}$, the invariant $w_{2}$ is killed by the $P^{1}$. Moreover, note that as the image of $\left[\Delta_{1, n}\right]$ is divisible by 2 but not by 4 , the intersection of the kernel of $A_{G}^{0}\left(\Delta_{1, n}, \mathrm{~K}_{2^{r}} Z\right) \rightarrow A_{G}^{1}\left(P^{n}, \mathrm{~K}_{2^{r}}\right)$ and the subring of constant invariants of $\mathrm{S}_{n-2}$ is exactly the 2-torsion.

On the other hand, the cokernel of the inclusion of $A_{G}^{0}\left(P^{n}, \mathrm{~K}_{2^{r}}\right)$ into the group $A_{G}^{0}\left(P_{\mathrm{sm}}^{n}, \mathrm{~K}_{2^{r}}\right)$ is isomorphic to the group of normalised invariants of $S_{n}$. Thus we have a map

$$
\operatorname{Inv}^{\bullet}\left(\mathrm{BS}_{n}, \mathrm{~K}_{2^{r}}\right) / \mathrm{K}_{2^{r}}^{\bullet}(k) \rightarrow \operatorname{Inv}^{\bullet}\left(\mathrm{BS}_{n-2}, \mathrm{~K}_{2^{r}}\right)_{2}
$$

which lowers degree by 1 . We claim this map is surjective. Let

$$
\alpha=\tau_{0}+\alpha_{1} \tau_{1}+\cdots+\alpha_{n / 2-1} \tau_{n / 2-1}
$$

with $\tau_{0}, \ldots, \tau_{n / 2-1} \in \mathrm{K}_{2^{r}}^{\bullet}(k)_{2}$ elements of $\operatorname{Inv}^{\bullet}\left(\mathrm{BS}_{n-2}, \mathrm{~K}_{2^{r}}\right)$. Let $\zeta_{1}, \ldots, \zeta_{n}$ be elements of $A_{G}^{0}\left(P_{\mathrm{sm}}^{n}, \mathrm{~K}_{2}\right)$ such that $\partial\left(\zeta_{1}\right)=1, \ldots, \partial\left(\zeta_{n}\right)=\alpha_{n / 2-1}$.

Then by Lemma 5.1 the element

$$
\zeta=\zeta_{1} \tau_{0}+\cdots+\zeta_{n / 2} \tau_{n / 2-1}
$$

belongs to $A_{G}^{0}\left(P_{\mathrm{sm}}^{n}, \mathrm{~K}_{2^{r}}\right)$. Now note that by the compatibility of the boundary map with the morphism of cycle modules $\mathrm{K}_{2^{r}} \rightarrow \mathrm{K}_{2}$, the restriction modulo 2 of $\partial\left(\zeta_{i}\right)$ is equal to $\alpha_{i-1}$. But then $\alpha_{i-1}-\partial\left(\zeta_{i}\right)$ is a multiple of 2 , proving that $\partial(\zeta)=\alpha$. This concludes the proof.

Corollary 7.7. Assume that $\ell$ is a power of 2 . If $g$ is even, the cohomological invariants with coefficients in $\mathrm{M}$ of $\left[P_{\mathrm{sm}}^{2 g+2} / G\right]$ are isomorphic to the cohomological invariants of $\mathrm{S}_{2 g+2}$. 
If $g$ is odd, the cohomological invariants with coefficients in $D$ of $\left[P_{\mathrm{sm}}^{2 g+2} / G\right]$ are a direct sum of the cohomological invariants of $\mathrm{S}_{2 g+2}$ and a copy of $\mathrm{M}^{\bullet}(k)_{2}$ [2] coming from $\mathrm{PGL}_{2}$.

Proof. Consider the exact sequence

$$
A_{G}^{0}\left(P^{n}, \mathrm{M}\right) \hookrightarrow A_{G}^{0}\left(P_{\mathrm{sm}}^{n}, \mathrm{M}\right) \stackrel{\partial}{\rightarrow} A_{G}^{0}\left(\Delta_{1}^{n}, \mathrm{M}\right) \subset A_{G}^{0}\left(P_{\mathrm{sm}}^{n-2} \times P^{1}, \mathrm{M}\right) .
$$

As in the Lemma 7.6, we proceed by induction on even $n$. If the result is true for $n-2$, we reduce to showing that the map

$$
\partial: \alpha_{1} \cdot \mathbf{M}^{\bullet}(k)_{2} \oplus \cdots \oplus \alpha_{n / 2} \cdot \mathbf{M}^{\bullet}(k)_{2} \rightarrow \mathbf{M}^{\bullet}(k)_{2} \oplus \cdots \oplus \alpha_{n / 2-1} \cdot \mathbf{M}^{\bullet}(k)_{2}
$$

is surjective. This is done exactly as in the Lemma 7.6, using the structure of an $A_{G}^{0}\left(P_{\mathrm{sm}}^{n}, \mathrm{~K}_{\ell}\right)$-module of $A_{G}^{0}\left(P_{\mathrm{sm}}^{n}, \mathrm{M}\right)$.

Lemma 7.8. Consider the line bundle $\mathcal{L}$ associated to the $\mathbb{G}_{m}$-torsor

$$
\mathcal{H}_{g} \rightarrow\left[P_{\mathrm{sm}}^{2 g+2} / G\right]
$$

The first Chern class $c_{1}(\mathcal{L})$ is equal to:

$\circ \lambda_{1}-t$ if $g$ is even.

$\circ t-2$ s if $g$ is odd.

Proof. The first formula is proven in [9, Lemma 3.2] and the second in [13, Eq. 3.2].

Theorem 7.9. Let $\ell$ be a power of 2. For all $g$, there is a submodule $\mathrm{N}_{g}^{\bullet}(k)$ of $\mathrm{M}^{\bullet}(k)_{2}$ such that the following are true:

1. If $g$ is even, there is an exact sequence

$$
0 \rightarrow \operatorname{Inv}^{\bullet}\left(\mathrm{BS}_{n}, \mathrm{M}\right) \rightarrow \operatorname{Inv}^{\bullet}\left(\mathcal{H}_{g}, \mathrm{M}\right) \rightarrow \mathrm{N}_{g}^{\bullet}(k) \rightarrow 0
$$

such that the inverse image of a nonzero element in $\mathrm{N}_{g}^{\bullet}(k)$ has degree at least $g+2$.

2. If $g$ is odd, let

$$
I_{g}=\alpha_{2} \cdot \mathbf{M}^{\bullet}(k)_{2} \oplus \cdots \oplus \alpha_{n} \cdot \mathbf{M}^{\bullet}(k)_{2} \subset \operatorname{Inv}^{\bullet}\left(\mathrm{BS}_{n}, \mathbf{M}\right)
$$

There is an exact sequence

$$
0 \rightarrow \mathrm{M}^{\bullet}(k)_{2} \oplus \mathrm{M}^{\bullet}(k)_{4}[1] \oplus I_{g} \oplus \mathrm{M}^{\bullet}(k)_{2}[2] \rightarrow \operatorname{Inv}^{\bullet}\left(\mathcal{H}_{g}, \mathrm{M}\right) \rightarrow \mathrm{N}_{g}^{\bullet}(k) \rightarrow 0
$$

such that the inverse image of a nonzero element in $\mathrm{N}_{g}^{\bullet}(k)$ has degree at least $g+2$. The $\mathrm{M}^{\bullet}(k)_{4}[1]$ is equal to $\alpha_{1}^{\prime} \cdot \mathbf{M}^{\bullet}(k)_{4}$, where $\alpha_{1}^{\prime}$ is a square root of $\alpha_{1}$.

Proof. The map $\mathcal{H}_{g} \rightarrow\left[P_{\mathrm{sm}}^{2 g+2} / G\right]$ is a $\mathbb{G}_{m}$-torsor. In particular, it is smooth Nisnevich, so the pullback on cohomological invariants is injective. We have to check whether there are invariants defined on $\mathcal{H}_{g}$ that do not come from the base.

Let $\mathcal{L} \rightarrow\left[P_{\mathrm{sm}}^{2 g+2} / G\right]$ be a line bundle determined to the $\mathbb{G}_{m}$-torsor: its equivariant Chow groups with coefficients are isomorphic to those of $\left[P_{\mathrm{sm}}^{2 g+2} / G\right]$. The inclusion of the zero section of $\mathcal{L}$ gives us the following long exact sequence:

$$
0 \rightarrow A_{G}^{0}\left(P_{\mathrm{sm}}^{2 g+2}, \mathrm{M}\right) \rightarrow \mathrm{Inv}^{\bullet}\left(\mathcal{H}_{g}, \mathrm{M}\right) \stackrel{\partial}{\rightarrow} A_{G}^{0}\left(P_{\mathrm{sm}}^{2 g+2}, \mathrm{M}\right) \stackrel{c_{1}(\mathcal{L})}{\longrightarrow} A_{G}^{1}\left(P_{\mathrm{sm}}^{2 g+2}, \mathrm{M}\right)
$$


Our goal is to understand the kernel of $c_{1}(\mathcal{L})$. We will do the computation in the odd-genus case. The even case is much easier and follows from the same reasoning.

In this case, we have $c_{1}(\mathcal{L})=t-2 s$. The submodule of $A_{G}^{1}\left(P_{\mathrm{sm}}^{2 g+2}, \mathrm{M}\right)$ generated by $t$ and $s$ multiplied by $\mathbf{M}^{\bullet}(k)$ has the single relation $(4 g+2) t=0$. Note that the relation implies that the submodule $t \cdot \mathrm{M}^{\bullet}(k)$ is of $2^{r}$ torsion and of $4 g+2$ torsion. As $2 g+1$ is odd, we get that the submodule $t \cdot \mathrm{M}^{\bullet}(k)$ is isomorphic to $\mathbf{M}^{\bullet}(k) / 2 \mathbf{M}^{\bullet}(k)$.

On the other hand, the submodule $s \cdot \mathrm{M}^{\bullet}(k)$ has no additional relation, so the annihilator of $2 s$ is exactly $\mathbf{M}^{\bullet}(k)_{2}$. Finally, it is easy to see that if $(t-2 s) \cdot \tau=0$ for some $\tau$ in $\mathbf{M}^{\bullet}(k)$, then both $t \cdot \tau=0$ and $2 s \cdot \tau=0$. This implies that any such $\tau$ must belong to both the 2-torsion of $\mathrm{M}^{\bullet}(k)$ and $2 \mathrm{M}^{\bullet}(k)-$ that is, $\tau$ is in $2 \cdot \mathbf{M}^{\bullet}(k)_{4}$.

Now let us pick $\mathrm{M}=\mathrm{K}_{4}$. The kernel of $c_{1}(\mathcal{L})$, restricted to the elements of cohomological degree 0 , is generated by 2 . Given an inverse image of 2 through $\partial$, we want to understand its relationship with the elements coming from $\left[P_{\mathrm{sm}}^{2 g+2} / G\right]$. We have $\operatorname{Inv}^{1}\left(\mathcal{H}_{g}, \mathrm{~K}_{4}\right)=\mathrm{H}^{1}\left(\mathcal{H}_{g}, \mu_{4}\right)$. The latter has to surject onto Pic $\left(\mathcal{H}_{g}\right)_{4}=\mathbb{Z} / 4 \mathbb{Z}$. Comparing the two formulas, we conclude that up to elements coming from the base field, there must be an element $\alpha_{1}^{\prime} \in \operatorname{Inv}^{1}\left(\mathcal{H}_{g}, \mathrm{~K}_{4}\right)$ such that $2 \alpha_{1}^{\prime}=\alpha_{1}$ and $\partial \alpha_{1}^{\prime}=2$.

For $\mathrm{M}$ an arbitrary $2^{r}$-torsion cycle module, we can consider the submodule $\alpha_{1}^{\prime} \cdot \mathrm{M}^{\bullet}(k)_{4}$. It is immediate that the map $\partial$ sends it surjectively to $2 \cdot \mathbf{M}^{\bullet}(k)_{4}$ with kernel given exactly by $\alpha_{1} \cdot \mathbf{M}^{\bullet}(k)_{2}$.

Now consider an element

$$
\alpha=\tau_{0}+\alpha_{1} \tau_{1}+\cdots+\alpha_{g} \tau_{g}+w_{2} \sigma \in A_{G}^{0}\left(P_{\mathrm{sm}}^{2 g+2}, \mathrm{M}\right) .
$$

We want to show that if $c_{1}(\mathcal{L}) \alpha=0$, then we must have $\tau_{1}=\cdots=\tau_{g}=\sigma=0$. Note that the highestdegree element $\alpha_{g+1}$ does not appear in the formula. Recall also that every $\tau_{i}$ is of 2-torsion for $i>0$.

If $c_{1}(\mathcal{L}) \alpha=0 \in A_{G}^{1}\left(P_{\mathrm{sm}}^{n}, \mathrm{M}\right)$, then the pullback of this element to the nonequivariant group $A^{0}\left(P_{\mathrm{sm}}^{n}, \mathrm{M}\right)$ must be 0 as well. The pullback of $c_{1}(\mathcal{L})$ is equal to $t$, and $w_{2}$ goes to 0 , so we get $t\left(\tau_{0}+\alpha_{1} \tau_{1}+\cdots+\alpha_{g} \tau_{g}\right)=0$.

For every $n \geq 4$ even, consider the morphism

$$
\Phi_{n}: A^{0}\left(P_{\mathrm{sm}}^{n}, \mathrm{M}\right) \stackrel{\partial}{\rightarrow} A^{0}\left(\Delta_{[1], n}, \mathrm{M}\right) \stackrel{\pi_{*}^{-1}}{\longrightarrow} A^{0}\left(P_{\mathrm{sm}}^{n-2} \times P^{1}, \mathrm{M}\right) \simeq A^{0}\left(P_{\mathrm{sm}}^{n-2}, \mathrm{M}\right)
$$

where $\pi: P_{\mathrm{sm}}^{n-2} \times P^{1} \rightarrow \Delta_{[1], n}$ maps $(f, g)$ to $f g^{2}$ and the last isomorphism is due to the projective bundle formula. By construction, we know that $\partial\left(\alpha_{i}\right)=\alpha_{i-1}+\beta$, where $\beta$ belongs to the submodule generated by $\alpha_{i-2}, \ldots, \alpha_{1}, 1$. In particular,

$$
\Phi_{2 g+2}(t \alpha)=t\left(\alpha^{\prime}+\alpha_{g-1} \tau_{g}\right)
$$

where $\alpha^{\prime}$ is a combination of multiples of $1, \ldots, \alpha_{g-2}$. After repeating this process $g-1$ times, we eventually end up with $t \tau_{g}=0$. As the image of $A^{0}\left(\Delta_{n}^{1}, \mathrm{M}\right)$ in $A^{1}\left(P^{n}, \mathrm{M}\right)$ is generated by the pushforward of 1 , which is an even multiple of $t$, there are no additional relations in the submodule $t \mathrm{M}^{\bullet}(k)_{2}$. This implies that $\tau_{g}=0$, thus $t\left(\tau_{0}+\alpha_{1} \tau_{1}+\cdots+\alpha_{g-1} \tau_{g-1}\right)=0$ in $A_{G}^{1}\left(P_{\mathrm{sm}}^{n}, \mathrm{M}\right)$.

Applying the same argument several times, we deduce $\tau_{i}=0$ for $i>0$ and $t \tau_{0}=0$, from which we also deduce that $\tau_{0}=2 \tau_{0}^{\prime}$. In other terms, we have proved that $\alpha=\tau_{0}+\sigma w_{2}$, hence

$$
0=(t-2 s)\left(\tau_{0}+w_{2} \sigma\right)=(-2 s+t) \tau_{0}+t w_{2} \sigma .
$$

Note that the submodule $t\left(w_{2} \cdot \mathrm{M}^{\bullet}(k)_{2}\right)$ has no additional relations and is independent from $t \mathrm{M}^{\bullet}(k)_{2}$ and $s \mathrm{M}^{\bullet}(k)$ due to the projection formula and the description of the cohomological invariants of $\mathrm{PGL}_{2}$. Consequently, we need for $(t-2 s) \tau_{0}$ and $t w_{2} \sigma$ to be separately zero. We already know that the first requirement is equivalent to $\tau_{0} \in 2 \mathrm{M}^{\bullet}(k)_{4}$, and the second implies $\sigma=0$. 
Finally, consider an element

$$
\alpha=\tau_{0}+\alpha_{1} \tau_{1}+\cdots+\alpha_{g} \tau_{g}+w_{2} \sigma+\alpha_{g+1} \tau_{g+1} .
$$

We may assume it is of homogeneous cohomological degree. If $c_{1}(\mathcal{L}) \alpha=0$, either $\tau_{g+1}=0$, in which case $\alpha=\tau_{0} \in 2 \mathbf{M}^{\bullet}(k)_{4}$, or $\tau_{g+1} \neq 0$. Then we can consider the map $\operatorname{Inv}^{\bullet}\left(\mathcal{H}_{g}, \mathbf{M}\right) \rightarrow \mathbf{M}^{\bullet}(k)_{2}$ given by composing $\partial$ with the map sending $\alpha \in A_{G}^{0}\left(P_{\mathrm{sm}}^{n}, \mathrm{M}\right)$ to $\tau_{g+1}$. The kernel of this map is exactly $\mathbf{M}^{\bullet}(k) \oplus \mathbf{M}^{\bullet}(k)_{4}[1] \oplus I_{g} \oplus \mathbf{M}^{\bullet}(k)_{2}[2]$, and the inverse image of a nonzero element in $\mathbf{M}^{\bullet}(k)_{2}$ has degree at least $g+2$. This concludes the proof.

Remark 7.10. Using the same techniques as in [8, Section 2.3], we can conclude that when $g$ is even, the module $\mathrm{N}_{g}^{\bullet}(k)$ is equal to $\mathbf{M}^{\bullet}(k)_{2}$ and explicitly describe the module structure:

$$
\operatorname{Inv}^{\bullet}\left(\mathcal{H}_{g}, \mathbf{M}\right)=\alpha_{1} \cdot \mathbf{M}^{\bullet}(k)_{4 g+2} \oplus I_{g} \oplus \mathbf{M}^{\bullet}(k)_{2}[g+2],
$$

where the last component is given by the element $\beta_{g+2}$ defined in [8, Section 2] and the Inv $\bullet\left(\mathcal{H}_{g}, \mathrm{~K}_{\ell}\right)$ module structure can be easily deduced from the multiplicative structure described in [8, Thm. 3.1].

When $g$ is odd, as explained in [8, Section 4], we can reach similar conclusions but only when $k$ is algebraically closed.

Remark 7.11. Let $\overline{\mathcal{H}}_{g}$ be the compactification of $\mathcal{H}_{g}$ by means of stable hyperelliptic curves. Then, following [8, Appendix A], we can easily conclude that the normalised cohomological invariants $\operatorname{Inv} \bullet\left(\overline{\mathcal{H}}_{g}, \mathrm{M}\right)$ are trivial whenever $\mathrm{M}$ is torsion.

\section{The Brauer group of $\mathcal{H}_{g}$}

We are finally ready to describe the Brauer group of $\mathcal{H}_{g}$. The following descriptions are immediate consequences of the description of $\operatorname{Inv}^{\bullet}\left(\mathcal{H}_{g}, \mathrm{M}\right)$ when $\mathrm{M}=\mathrm{H}_{\mu_{\ell}}$. Let $c=\operatorname{char}(k)$ be the characteristic of the base field, and let $r_{g}$ be the remainder of $g \bmod 2$. Define $\ell_{g}=\ell_{g}(c)$ as the largest divisor of $2^{r_{g}}(4 g+2)$ which is not divisible by $c$.

Theorem 8.1. We have

$$
{ }^{c} \operatorname{Br}\left(\mathcal{H}_{g}\right) \simeq{ }^{c} \operatorname{Br}(k) \oplus \mathrm{H}^{1}\left(k, \mathbb{Z} / \ell_{g} \mathbb{Z}\right) \oplus \mathbb{Z} / 2 \mathbb{Z}^{1+r_{g}} .
$$

Proof. From the description of $\operatorname{Inv} \bullet\left(\mathcal{H}_{g}, \mathrm{H}_{\mu_{\ell}^{\vee}}\right)$, it is clear that the cohomological Brauer group of $\mathcal{H}_{g}$, or its prime-to- $\operatorname{char}(k)$ part in positive characteristic, is of $\ell_{g}$-torsion. Then the formula for ${ }^{c} \mathrm{Br}^{\prime}$ follows immediately by taking $n=\ell_{g}$, and Theorem 2.1 assures us that every element comes from the Brauer group.

Now we want to explicitly describe the generators of ${ }^{c} \operatorname{Br}\left(\mathcal{H}_{g}\right)$. By the description in Theorem 8.1, an element in ${ }^{c} \operatorname{Br}\left(\mathcal{H}_{g}\right)$ must decompose as a sum of:

$\circ$ elements coming from the base field;

$\circ$ elements coming from the cup product of the cohomology of the base field and the degree 1

cohomological invariant, which by Lemmas 2.18 and 2.19 are all represented by cyclic algebras; and

$\circ$ the (one or two) copies of $\mathbb{Z} / 2 \mathbb{Z}$ coming from $\mathrm{BS}_{2 g+2}$ and $\mathrm{BPGL}_{2}$.

Differently from the case of $\mathcal{M}_{1,1}$, there are nonconstant elements which do not come from the the cup product

$$
\mathrm{H}^{1}\left(\mathcal{H}_{g}, \mu_{\ell_{g}}\right) \otimes \mathrm{H}^{1}\left(\mathcal{H}_{g}, \mathbb{Z} / \ell_{g} \mathbb{Z}\right) \rightarrow \mathrm{Br}\left(\mathcal{H}_{g}\right)
$$


namely the copies of $\mathbb{Z} / 2 \mathbb{Z}$. One way to see this is to note that when $k$ is algebraically closed these generators are still nonzero, but

$$
\mathrm{H}^{1}\left(\mathcal{H}_{g}, \mu_{\ell_{g}}\right)=\mathrm{H}^{1}\left(\mathcal{H}_{g}, \mathbb{Z} / \ell_{g} \mathbb{Z}\right)=\mathbb{Z} / \ell_{g} \mathbb{Z},
$$

and the cup product $\mathrm{H}^{1}\left(\mathcal{H}_{g}, \mu_{\ell_{g}}\right) \cdot \mathrm{H}^{1}\left(\mathcal{H}_{g}, \mathbb{Z} / \ell_{g} \mathbb{Z}\right)$ is 0 , as given a generator $\gamma$ of $\operatorname{Inv}^{1}\left(\mathcal{H}_{g}, \mathbb{Z} / \ell_{g} \mathbb{Z}\right)$, we have $\gamma \cdot \gamma=\{-1\} \gamma=0$.

We now proceed to give a more detailed depiction of the elements in ${ }^{c} \operatorname{Br}\left(\mathcal{H}_{g}\right)$ that come from neither the base field nor the cup product.

Start by considering the symmetric group $\mathrm{S}_{2 g+2}$. From the work of Schur [25] we know that there exists a group $\hat{\mathrm{S}}_{2 g+2}$ which is a nonsplit extension of $\mathrm{S}_{2 g+2}$ by $\mu_{2}-$ that is, there is a nonsplit exact sequence of groups

$$
1 \longrightarrow \mu_{2} \longrightarrow \hat{S}_{2 g+2} \longrightarrow S_{2 g+2} \longrightarrow 1
$$

The induced morphism of classifying stacks makes $\mathrm{BS}_{2 g+2}$ into a gerbe over $\mathrm{BS}_{2 g+2}$, banded by $\mu_{2}$. This gerbe cannot be a trivial $\mu_{2}$-gerbe, as otherwise there would exist a homomorphism $\mathrm{S}_{2 g+2} \rightarrow \hat{\mathrm{S}}_{2 g+2}$ splitting the short exact sequence given. In particular, $B \hat{S}_{2 g+2}$ is not trivial over any algebraically closed field.

The cohomology group $\mathrm{H}^{2}\left(\mathrm{BS}_{2 g+2}, \mu_{2}\right)$ is isomorphic to the group of gerbes on $\mathrm{BS}_{2 g+2}$ banded by $\mu_{2}$. This, together with Theorem 2.1, lets us regard $\left[\mathrm{BS}_{2 g+2}\right]$ as a nontrivial element of the Brauer group of $\mathrm{BS}_{2 g+2}$. It easily follows from Proposition 5.10 that ${ }^{c} \mathrm{Br}\left(\mathrm{BS}_{2 g+2}\right) \simeq \mathbb{Z} / 2 \mathbb{Z}$, hence $\left[\mathrm{BS}_{2 g+2}\right]$ is actually a generator of this Brauer group. Moreover, $\left[\mathrm{BS}_{2 g+2}\right]$, this time regarded as an Azumaya algebra, cannot be a cyclic algebra, as otherwise the gerbe $\mathrm{BS}_{2 g+2}$ should be trivial over an algebraically closed field

Let $\hat{\mathcal{H}}_{g}$ be the $\mu_{2}$-gerbe on $\mathcal{H}_{g}$ obtained by pulling back $\mathrm{B} \hat{S}_{2 g+2}$ along the classifying morphism $\mathcal{H}_{g} \rightarrow \mathrm{BS}_{2 g+2}$. Then the computation contained in Theorem 8.1 implies that $\left[\hat{\mathcal{H}}_{g}\right]$ induces a generator of $\mathbb{Z} / 2 \mathbb{Z}^{2}$ complementary to $\left[\mathrm{B} \hat{S}_{2 g+2}\right]$ in ${ }^{c} \operatorname{Br}\left(\mathcal{H}_{g}\right)$.

Here is an alternative description of this generator as an equivalence class of a Severi-Brauer variety: by [25] there exists a projective representation $\mathbb{P}(V)$ of $S_{2 g+2}$ which does not lift to a linear representation $V$. Then the equivalence class of the Severi-Brauer variety $\left[\mathbb{P}(V) / \mathrm{S}_{2 g+2}\right]$ over $\mathrm{BS}_{2 g+2}$ is a nontrivial element of ${ }^{c} \mathrm{Br}\left(\mathrm{BS}_{2 g+2}\right) \simeq \mathbb{Z} / 2 \mathbb{Z}$, hence a generator.

Again by Theorem 8.1, this implies that the Severi-Brauer variety over $\mathcal{H}_{g}$ defined as $\mathcal{H}_{g} \times_{\mathrm{BS}_{2 g+2}}$ $\left[\mathbb{P}(V) / S_{2 g+2}\right]$ generates a copy of $\mathbb{Z} / 2 \mathbb{Z}$ in ${ }^{c} \operatorname{Br}\left(\mathcal{H}_{g}\right)$.

We now turn to the second copy of $\mathbb{Z} / 2 \mathbb{Z}$ in ${ }^{c} \operatorname{Br}\left(\mathcal{H}_{g}\right)$, which appears only when $g$ is odd. Regarding ${ }^{c} \operatorname{Br}\left(\mathcal{H}_{g}\right)$ as the group of equivalence classes of Severi-Brauer varieties, it is immediate to check that a generator for the copy of $\mathbb{Z} / 2 \mathbb{Z}$ is given by the universal conic $\mathcal{C} \rightarrow \mathcal{H}_{g}$, which is the quotient of the universal hyperelliptic curve by the universal hyperelliptic involution.

Indeed, the universal conic induces the classifying morphism $\mathcal{H}_{g} \rightarrow \mathrm{BPGL}_{2}$, which we use to pull back the degree 2 cohomological invariant $w_{2}$ of $\mathrm{BPGL}_{2}$.

Observe that such a universal conic exists also when $g$ is even, but in this case it is the projectivisation of a vector bundle, hence its class is 0 in the Brauer group.

Acknowledgements. The idea for this paper originated from a question posed to the second author by R. Fringuelli. We thank him for asking the right question at the right time. We are thankful to David Rydh for helpful discussions and a very careful reading of a preliminary draft of the paper, and to Burt Totaro, Zinovy Reichstein and Angelo Vistoli for helpful suggestions. Finally, we are grateful to the anonymous referee for a very thorough and useful report.

We acknowledge support by the German Research Foundation (DFT) and the Open Access Publication Fund of HumboldtUniversität zu Berlin. 


\section{References}

[1] B. Antieau and L. Meier, 'The Brauer group of the moduli stack of elliptic curves', to appear in Algebra Number Theory. 14(9) (2020), 2259-2333.

[2] A. Arsie and A. Vistoli, 'Stacks of cyclic covers of projective spaces', Compos. Math. 140(3) (2004), 647-666.

[3] M. Bolognesi and A. Vistoli, 'Stacks of trigonal curves', Trans. Amer. Math. Soc. 364(7) (2012), 3365-3393.

[4] A. de Jong, 'A result of Gabber', Preprint. URL: https://www.math.columbia.edu/dejong/papers/2-gabber.pdf.

[5] A. Di Lorenzo, 'The Chow ring of the stack of hyperelliptic curves of odd genus', Int. Math. Res. Not. (2021), no. 4, 2642-2681.

[6] A. Di Lorenzo, 'Cohomological invariants of the stack of hyperelliptic curves of odd genus', Transform. Groups 26(1) (2021) 165-214.

[7] A. Di Lorenzo, 'Integral Picard group of the stack of quasi-polarized K3 surfaces of low degree', Preprint, 2019, arXiv: 1910.08758.

[8] A. Di Lorenzo and R. Pirisi, 'A complete description of the cohomological invariants of even genus hyperelliptic curves', Doc. Math. 26 (2021), 199-230.

[9] D. Edidin and D. Fulghesu, 'The integral Chow ring of the stack of hyperelliptic curves of even genus', Math. Res. Lett. 16(1) (2009), 27-40.

[10] D. Edidin and W. Graham, 'Equivariant intersection theory', Invent. Math. 131(3) (1998), 595-634.

[11] D. Edidin, B. Hassett, A. Kresch and A. Vistoli, 'Brauer group and quotient stacks', Amer. J. Math. 123(4) (2001), $761-777$.

[12] R. Fringuelli and R. Pirisi, 'The Brauer group of the universal moduli space of vector bundles over smooth curves', Int. Math. Res. Not. IMRN, published online at https://doi.org/10.1093/imrn/rnz300.

[13] D. Fulghesu and F. Viviani, 'The Chow ring of the stacks of cyclic covers of the projective line', Ann. Inst. Fourier (Grenoble) 61(6) (2011), 2249-2275.

[14] O. Gabber, 'Some theorems on Azumaya algebras', in The Brauer group, (Sem. Les plans-sur-Bex, 1980), Lecture Notes in Mathematics vol. 844 ( Springer, Berlin, 1981), 129-209.

[15] S. Garibaldi, A. Merkurjev and J.-P. Serre, Cohomological Invariants in Galois Cohomology, University Lecture Series vol. 28 ( American Mathematical Society, Providence, RI, 2003).

[16] S. Gille and C. Hirsch, 'On the splitting principle for cohomological invariants of reflection groups', Transform. Groups, Available online at https://doi.org/10.1007/s00031-020-09637-6.

[17] P. Guillot, 'Geometric methods for cohomological invariants', Doc. Math. 12 (2007), PP-PP.

[18] A. Kresch and A. Vistoli, 'On coverings of Deligne-Mumford stacks and surjectivity of the Brauer map', Bull. Lond. Math. Soc. 36(2) (2004), 188-192.

[19] M. Lieblich, 'Period and index in the Brauer group of an arithmetic surface', J. Reine Angew. Math. 669 (2011), 1-41. With an appendix by D. Krashen.

[20] L. Meier, 'Computing Brauer groups via coarse moduli', preprint (2018) URL: http://www.staff.science. uu.nl/meier007/CoarseBrauer.pdf.

[21] R. Pirisi, 'Cohomological invariants of hyperelliptic curves of even genus', Algebr. Geom. 4(4) (2017), 424-443.

[22] R. Pirisi, 'Cohomological invariants of algebraic stacks', Trans. Amer. Math. Soc. 370(3) (2018), 1885-1906.

[23] R. Pirisi, 'Cohomological invariants of hyperelliptic curves of genus 3', Doc. Math. 23 (2018), 969-996.

[24] M. Rost, 'Chow groups with coefficients', Doc. Math. 1(16) (1996), 319-393.

[25] J. Schur, 'Über die Darstellung der symmetrischen und der alternierenden Gruppe durch gebrochene lineare Substitutionen, J. Reine Angew. Math. 139 (1911), 155-250.

[26] M. Shin, 'The Brauer group of the moduli stack of elliptic curves over algebraically closed fields of characteristic 2', J. Pure Appl. Algebra 223(5) (2019), 1966-1999.

[27] The Stacks-Project authors, Stacks-Project, 2018, URL: https://stacks.math.columbia.edu/.

[28] B. Totaro, 'The Chow ring of a classifying space', in Algebraic K-Theory, Proceedings of Symposia in Pure Mathematics vol. 67 (Amer. Math. Soc., Providence, RI, 1999), 259-281.

[29] V. Voevodsky, ' $n$ Motivic cohomology with Z/ $\ell$-coefficients', Ann. of Math. (2) 174 (2011), 401-438. 